\title{
Mutational Analysis of Mitochondrial tRNA Genes in 200 Patients with Type 2 Diabetes Mellitus
}

\author{
Liangyan Lin' \\ Dongdong Zhang' \\ Qingsong Jin' \\ Yaqin Teng' \\ Xiaoyan Yao' \\ Tiantian Zhao' \\ Xinmiao $X^{2}$ \\ Yongjun Jin'
}

'Department of Endocrinology and Metabolism, Yantai Affiliated Hospital of Binzhou Medical University, Yantai, Shandong, People's Republic of China; ${ }^{2}$ Department of Endocrinology, Yantai Yeda Hospital, Yantai, Shandong, People's Republic of China
Correspondence: Yongjun Jin

Department of Endocrinology and Metabolism, Yantai Affiliated Hospital of Binzhou Medical University, Jinbu Street No. 717, Yantai, 264100, People's

Republic of China

$\mathrm{Tel} / \mathrm{Fax}+86535-4770613$

Email jinyj000I@sina.com
Objective: Previous studies showed that variants in mitochondrial DNA (mtDNA) are associated with type 2 diabetes mellitus (T2DM). However, the relationships between mitochondrial tRNA (mt-tRNA) variants and T2DM remain poorly understood.

Methods: In this study, we performed a mutational screening of $22 \mathrm{mt}$-tRNA genes in a cohort of 200 Han Chinese subjects with T2DM and 200 control subjects through PCRSanger sequencing. The identified mt-tRNA variants were assessed for their pathogenicity via the phylogenetic approach, structural and functional analysis. Furthermore, two Han Chinese pedigrees with maternally inherited diabetes and deafness (MIDD) were reported by clinical and genetic assessments.

Results: A total of 49 genetic variants in mt-tRNA genes were identified; among them, 31 variants (17 pathogenic/likely pathogenic) were absent in controls, located at extremely conserved nucleotides, may have potential structural and functional significance, thereby considered to be T2DM-associated variants. In addition, sequence analysis of entire mitochondrial genomes of the matrilineal relatives from two MIDD pedigrees revealed the occurrence of tRNA $^{\text {Leu(UUR) }}$ A3243G and T3290C mutations, as well as sets of polymorphisms belonging to mitochondrial haplogroups F2 and D4. However, the lack of any functional variants in connexin 26 gene (GJB2) and tRNA 5-methylaminomethyl-2-thiouridylate (TRMU) suggested that nuclear genes may not play active roles in clinical expression of MIDD in these pedigrees.

Conclusion: Our data indicated that mt-tRNA variants were associated with T2DM, screening for mt-tRNA pathogenic mutations was recommended for early detection and prevention of mitochondrial diabetes.

Keywords: type 2 diabetes mellitus, mitochondrial tRNA, variants, Chinese population

\section{Introduction}

Diabetes is a very complex disease characterized by the presence of chronic hyperglycemia. Clinically, insulin-dependent type 1 and non-insulin-dependent type 2 are the main types of diabetes. Among them, type 2 diabetes mellitus (T2DM, [MIM125853]) is a common endocrine disorder affecting approximately $10 \%$ of adult population. ${ }^{1}$ In most cases, T2DM exhibits high blood glucose in the context of insulin resistance (IR) and relative insulin deficiency. To date, the etiology of T2DM is still undetermined. Now it has been recognized that this disorder can be caused by some acquired factors, including inherited genetic factors or the interactions between genetic and environmental factors. ${ }^{2}$ In particular, maternally inheritance has been observed in some pedigrees, highlighting the contributions of mitochondrial DNA (mtDNA) mutations to T2DM. ${ }^{3}$ Since the 
landmark discovery of the association between mitochondrial diabetes and 10.4-kb deletion in mtDNA, ${ }^{4}$ a growing number of T2DM-associated mtDNA mutations have been identified. Most of them are located at mitochondrial tRNA (mt-tRNA) genes. As adapter molecules to convert the genetic codes into amino acid sequences, mt-tRNAs play central roles in mitochondrial protein synthesis, as well as maintenance of respiratory chain functions. ${ }^{5}$ By molecular level, almost every mt-tRNA has a highly conserved cloverleaf structure, consisting of Acceptor arm, DHU-Loop, anticodon stem, variable region, and $\mathrm{T} \psi \mathrm{C}$ loop, with an average length of 73 nucleotides. Although mt-tRNAs comprise only around $10 \%$ of the total coding capacity of mtDNA genes, more than half of mtDNA mutations causing diseases are located in mt-tRNA genes, as indicated in Mitomap database (https://www. mitomap.org/MITOMAP) ${ }^{6}{ }^{6}$ emphasizing the importance of mt-tRNAs for mitochondrial function.

Recent experimental studies have suggested that tRNA $^{\text {Leu(UUR) }}$ A3243G and T3264C, tRNA ${ }^{\text {Gly }}$ T10003C, tRNA $^{\text {Glu }}$ T14709C, and tRNA ${ }^{\text {Thr }}$ G15897A are potential pathogenic mutations affecting T2DM predisposition. ${ }^{7-11}$ In particular, the well-known A3243G mutation affects the processing of mitochondrial RNA precursors, ${ }^{12}$ and base modification of this tRNA. ${ }^{13}$ In cytoplasmic hybrids (cybrids) harboring the $\mathrm{A} 3243 \mathrm{G}$ mutation, the level of aminoacylated

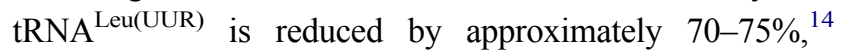
thereby influencing the protein synthesis and mitochondrial respiratory chain function. Furthermore, the T3290C mutation is localized at very conserved nucleotide of T $\psi \mathrm{C}$ loop in tRNA $^{\text {Leu(UUR) }}$, and is important for the steady-state level of tRNA $^{\text {Leu(UUR) }}$. Thus, these diabetes-related mtDNA mutations or variants, which are often presented in homoplasmy, exhibit a variability of clinical phenotypes. ${ }^{15}$ However, the pathophysiology of mt-tRNA mutations/variants in phenotypic manifestation of T2DM remains poorly understood.

In the current study, with the aim of exploring the T2DM-related mt-tRNA mutations/variants, 200 patients who have been diagnosed with T2DM, together with 200 age- and gender-matched controls, underwent the mutational screening of 22 mt-tRNA genes. As a result, a total of 49 genetic variants on 22 mt-tRNA genes are identified in this study. By the pathogenic evaluations including the phylogenetic analysis, potential structural and functional alternations, 17 T2DM-associated tRNA variants in 23 patients are identified. Furthermore, we perform clinical, genetic and molecular evaluations of two maternally inherited diabetes and deafness (MIDD) families carrying tRNA ${ }^{\text {Leu(UUR) }}$ A3243G and T3290C variants. To understand the contributions of mtDNA genetic background and nuclear genes to MIDD, we initiated the mutational screening of the entire mitochondrial genomes, as well as GJB2, TRMU from the matrilineal relatives in these pedigrees.

\section{Methods}

\section{Study Population}

In this case-control study for mutational screening of T2DMrelated mt-tRNA variants, a total of 200 genetically unrelated subjects who were diagnosed with T2DM, participating for this study; moreover, 200 control subjects who came from the Healthy Examination Center of our hospital were also recruited. This study was approved by the Ethics Committee of Yantai Affiliated Hospital of Binzhou Medical University, and each participant provided their written informed consent.

The diagnosis of T2DM was based on the criteria proposed by American Diabetes Association: ${ }^{16}$ (1) a fasting plasma glucose (FPG) level $\geq 7.0 \mathrm{mmol} / \mathrm{L}$; (2) a $2 \mathrm{~h}$ plasma glucose level after 75 -g oral glucose tolerance test (OGTT) $\geq 11.1 \mathrm{mmol} / \mathrm{L}$; (3) the level of Hemoglobin A1c $(\mathrm{HbA} 1 \mathrm{c}) \geq 6.5 \%$.

\section{Mutational Analysis of Mt-tRNA Genes}

Genomic DNA of each subject was extracted from the peripheral blood by using QIAamp Blood Kit (QIAGEN, Hilden, Germany). To detect T2DM-related mt-tRNA variants, polymerase chain reaction (PCR) was used to amplify fragments of all mt-tRNA genes in these subjects with appropriate primers, as described previously. ${ }^{17}$ For the subjects carrying the putative variants in mt-tRNA genes, fragments spanning the remaining regions of mtDNA genes were PCR amplified and sequenced to define the mtDNA haplogroups, according to a method described in a previous study. ${ }^{18}$ The PCR products were purified and sequenced by ABI 3730 DNA automatic sequencer (Applied Biosystems, Darmstadt, Germany). ${ }^{19}$ The sequence data were compared with the revised Cambridge reference sequence (rCRS, GenBank Accessible Number: NC_012920.1) using DNA STAR software package version 5.01 (DNASTAR Inc., Madison, USA) to detect the nucleotides alternations. ${ }^{20}$

\section{Analysis of Conservation Index $(\mathrm{Cl})$}

To further assess the pathogenic roles of mt-tRNA variants, the phylogenetic analysis was performed. Briefly, 17 
species were used for conservation analysis as described in a previous investigation. ${ }^{21}$ These species included Bos taurus, Cebus albifrons, Colobus guereza, Gorilla gorilla, Homo sapiens, Hylobates lar, Lemur catta, Macaca mulatta, Macaca sylvanus, Mus musculus, Nycticebus coucang, Pan paniscus, Pan troglodytes, Pongo pygmaeus, Pongo abelii, Papio hamadryas, and Tarsius bancanus. The CI was then calculated by comparing the human mtDNA variants with other 16 species. Notably, $\mathrm{CI} \geq 75 \%$ was regarded as having functional potential. ${ }^{22}$

\section{Structural Analysis}

The published secondary structures for the mt-tRNAs were used to define the stem and loop structure. ${ }^{23,24}$

\section{Characterization of Two Han Chinese Pedigrees Harboring Mt-tRNA ${ }^{\text {Leu(UUR) }}$ Variants}

As shown in Figure 1, two Han Chinese families with MIDD were ascertained in Yantai Affiliated Hospital of Binzhou Medical University. To identify personal or family histories of T2DM and other clinical abnormalities, all participants were interviewed and evaluated by physical examinations. For laboratory analysis, each participant's blood sample was collected between 7:00 AM and 10:00 AM after an overnight fast. The level of HbA1c was measured by using high-performance liquid chromatography (HPLC, Bio-Rad, CA, USA). In addition, the OGTT was performed by measuring the 0 and $2 \mathrm{~h}$ of plasma glucose concentrations after glucose administration.

Furthermore, the age-appropriate audiological examination of hearing loss was performed as described previously, $^{25}$ including pure-tone audiometry (PTA), auditory brainstem response, acoustic immittance measurement and distortion product otoacoustic emission. The PTA was calculated from the sum of the audiometric thresholds at 500, 1000, 2000, 4000 and $8000 \mathrm{~Hz}$. The severity of hearing loss was classified into 5 grades: normal $<26$ Decibel $(\mathrm{dB})$; mild $=26-40 \mathrm{~dB}$; moderate $=41-$ $70 \mathrm{~dB}$; severe $=71-90 \mathrm{~dB}$ and profound $>90 \mathrm{~dB}$, as suggested previously. ${ }^{26}$ The blood pressure (BP) was measured by an electronic sphygmomanometer and repeated for 3 times. Hypertension was defined according to the guidelines of the Joint National Committee on Detection, Evaluation and Treatment of High Blood Pressure (JNC VI), as a systolic $\mathrm{BP} \geq 140 \mathrm{mmHg}$ or the diastolic $\mathrm{BP} \geq 90$ mmHg. ${ }^{27}$

\section{Screening for the Entire Mitochondrial Genome Variants}

The complete mitochondrial genomes of the matrilineal individuals from two MIDD pedigrees (DM-101: II-3, II6 and III-5; DM-102: II-5, II-8, II-10 and III-7) were PCR amplified by using 24 primers, as previously described. ${ }^{28}$ The PCR products were purified and subsequently sequenced by ABI 3730 DNA automatic sequencer (Applied Biosystems, Darmstadt, Germany). The sequence data was then compared with the rCRS (GenBank Accessible Number: NC_012920.1) using DNA STAR software package version 5.01 (DNASTAR Inc., Madison, USA) to detect the nucleotides alternations. ${ }^{20}$

\section{Determining the Mitochondrial Haplogroups}

The classification of the mitochondrial haplogroups was based on the phylogenetic tree by Kong et al. ${ }^{29}$
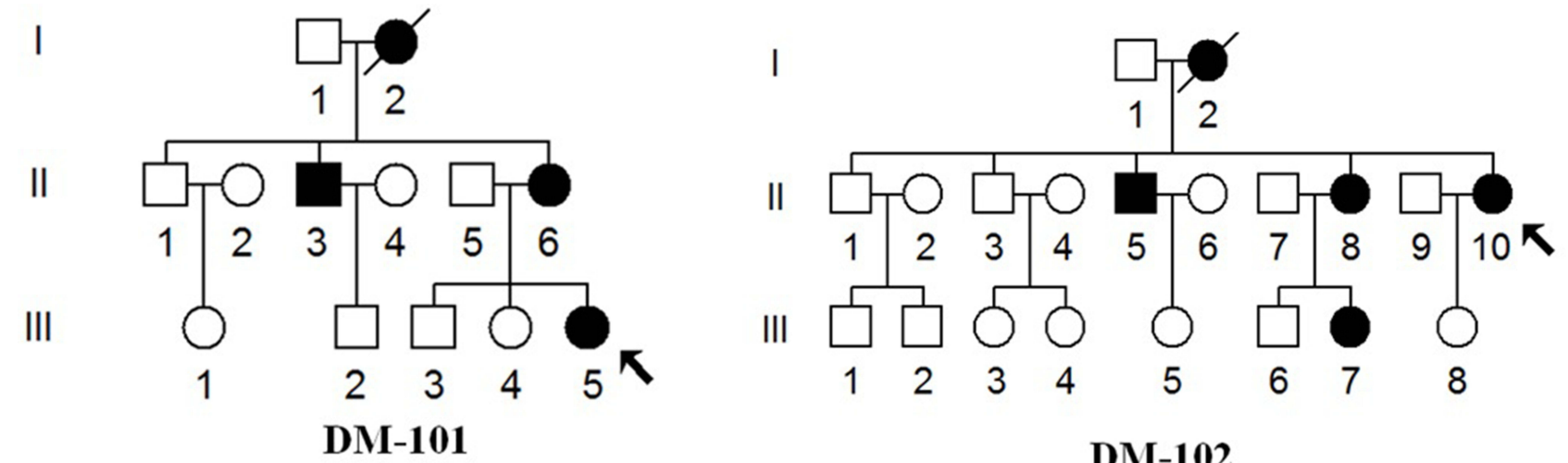

DM-102

Figure I Two Han Chinese pedigrees with MIDD, affected individuals are indicated by filled symbols. Arrows indicate the probands. 


\section{Mutational Screening for GJB2 Gene}

To see the contributions of GJB2 variants to clinical expression of MIDD, we conducted a mutational screening by using PCR amplification of the exons of GJB2 gene in matrilineal individuals (DM-101: II-3, II-6 and III-5; DM102: II-5, II-8, II-10 and III-7), the primer sequence for amplification of GJB2 gene were: forward-5'-TATGACA CTCCCCAGCACAG-3', and reverse-5'-GGGCAATGCT TAAACTGGC- $3^{\prime}{ }^{30}$ After PCR amplification and direct Sanger sequence analysis, the data were compared with the wild-type versions of GJB2 sequence (GenBank Accessible Number: M86849) to identify mutations/ variants. $^{30}$

\section{Analysis of TRMU Variants}

Previous study indicated that TRMU A10S variant may contribute to the phenotypic manifestation of deafnessassociated $12 \mathrm{~S}$ rRNA mutations. ${ }^{31}$ To understand the role of $T R M U$ in hearing impairment, the TRMU A10S variant was screened in the matrilineal relatives (DM-101: II-3, II6 and III-5; DM-102: II-5, II-8, II-10 and III-7) by using PCR amplification of exon 1 of this gene. The primer sequences for TRMU exon 1 were: forward-5'-ACAGCGC AGAAGAAGAGCAGT-3', and reverse-5'-ACAACGCCA CGACGGACG-3'. After PCR amplification and direct Sanger sequence, the data were compared with the wildtype versions of TRMU (GenBank Accessible Number: AF448221) to detect the variants. ${ }^{31}$

\section{Results}

\section{Clinical Characterization of 200 Patients with T2DM}

A total of 200 T2DM patients (117 females and 83 males) were recruited in this study. The age of these patients ranged from 30 to 68 years, with a median age of 42 years. The age at the onset of T2DM varied from 28 to 66 years, with an average of 40 years. Furthermore, 200 Han Chinese controls from the same region were healthy individuals and did not have any family history of T2DM, deafness, cardiovascular diseases or mitochondrial disorders. The age of these controls varied from 25 to 53 years, with an average of 38 years.

\section{Screening for Mt-tRNA Variants}

By sequencing 22 mt-tRNA genes in T2DM subjects and controls, we were able to identify 49 nucleotide alternations, as shown in Table 1. Among these, 3 variants were identified in tRNA ${ }^{\text {Phe }}, 2$ variants in $\mathrm{tRNA}^{\mathrm{Val}}, 2$ variants in tRNA $^{\text {Leu(UUR) }}, 2$ variants in tRNA $^{\text {Gln }}, 3$ variants in tRNA $^{\text {Met }}, 1$ variant in $\mathrm{tRNA}^{\text {Trp }}, 2$ variants in $\mathrm{tRNA}^{\mathrm{Ala}}, 3$ variants in tRNA ${ }^{\text {Cys, }} 1$ variant in tRNA ${ }^{\text {Asp }}, 3$ variants in tRNA $^{\text {Ser(UCN) }}, 2$ variants in tRNA ${ }^{\text {Lys }}, 2$ variants in tRNA ${ }^{\text {Gly }}$, 2 variants in tRNA ${ }^{\mathrm{Leu}(\mathrm{CUN})}, 2$ variants in tRNA ${ }^{\mathrm{Arg}}, 3$ variants in tRNA ${ }^{\text {His }}, 2$ variants in tRNA ${ }^{\text {Ser(AGY) }}, 1$ variant in tRNA $^{\text {Glu }}, 9$ variants in $\mathrm{tRNA}^{\mathrm{Thr}}$ and 2 variants in tRNA ${ }^{\text {Pro }}$.

\section{Assessment of the Pathogenicity}

We used the following criteria to evaluate the potential pathogenicity of mt-tRNA variants: (1) occurred in $<1 \%$ in the control subjects; (2) $\mathrm{CI} \geq 75 \%$, consistent with the evolutionary conservation at a given locus, as proposed by Ruiz-Pesini and Wallace; ${ }^{32}$ (3) the variants were predicted to alter the structure or function of tRNA molecules. As shown in Table 1, among them, the CIs of 22 variants were $\geq 75 \%$, the CIs of 15 variants were between $50 \%$ and $75 \%$, and the CIs of the remaining variants were $<50 \%$. Next, we used the secondary structure of mt-tRNAs to localize each variant with either a stem or a loop, in order to test whether the base changes altered the classic Watson-Crick base-pairing. We noticed that there were 13 variants occurring in the Acceptor arm, 12 variants in DHU-Loop, 5 variants in variable region, 12 variants in $\mathrm{T} \psi \mathrm{C}$ loop, 1 variant in ACC Terminus. Notably, 12 variants disrupted the Watson-Crick base-pairings, whereas 6 variants created novel Watson-Crick base-pairings. This analysis suggested that $17 \mathrm{mt}$-tRNA variants, which were well conserved and not detected in control subjects, may be pathogenic/likely pathogenic mutations (Table 1). These variants were as follows: tRNA ${ }^{\text {Leu(UUR) }} \mathrm{A} 3243 \mathrm{G}$ and T3290C, tRNA ${ }^{\text {Met }}$ A4435G and C4467A, tRNA ${ }^{\text {Trp }}$ A5514G, tRNA ${ }^{\text {Ala }}$ T5587C and A5655G, tRNA ${ }^{\text {Ser(UCN) }}$ C7502T and T7505C, tRNA ${ }^{\text {Lys }}$ G8313A, tRNA ${ }^{\text {Gly }}$ T10003C and A10055G, tRNA ${ }^{\text {Leu(CUN) }}$ A12308G and A12330G, tRNA $^{\text {Thr }}$ G15897A, A15924G and G15927A. Whereas other 32 variants were classified as "neutral polymorphisms" that were not well conserved or presented $>1 \%$ in control groups.

As shown in Figure 2, among 17 pathogenic/likely pathogenic variants, 5 of them occurred at Acceptor arm including $\mathrm{A} 4467 \mathrm{C}$ in $\mathrm{tRNA}^{\mathrm{Met}}$, A5514G in tRNA ${ }^{\text {Trp }}$, $\mathrm{A} 5655 \mathrm{G}$ in tRNA $^{\mathrm{Ala}}$, A10055G in tRNA $^{\text {Gly }}$ and A12330G in tRNA ${ }^{\text {Leu(CUN) }}$, abolished the classic WatsonCrick base-pairings of corresponding tRNAs. In addition, variants T7505C and C7502T in tRNA ${ }^{\text {Ser(UCN) }}$, G8313A in tRNA $^{\text {Lys }}, T_{10003 C}$ in tRNA ${ }^{\text {Gly }}$ and G15897A in tRNA ${ }^{\text {Thr }}$ 


\begin{tabular}{|c|c|c|c|c|c|c|c|c|c|c|c|c|c|c|c|c|c|c|c|c|c|c|c|}
\hline 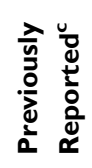 & & $\stackrel{\tilde{\nu}}{\tilde{y}}$ & $\stackrel{\tilde{y}}{\nu}$ & 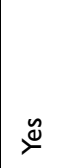 & $\stackrel{\mathscr{y}}{\check{\nu}}$ & $\stackrel{\mathscr{y}}{\check{\nu}}$ & 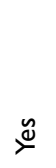 & $\stackrel{\mathscr{\nu}}{\check{\nu}}$ & $\stackrel{\mathscr{\nu}}{\check{\nu}}$ & $\stackrel{\mathscr{\Perp}}{\succ}$ & $\stackrel{\mathscr{\nu}}{\check{\nu}}$ & 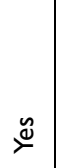 & 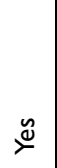 & $\stackrel{\tilde{\nu}}{\sim}$ & $\stackrel{\mathscr{\nu}}{\check{\nu}}$ & $\stackrel{\tilde{y}}{\tau}$ & 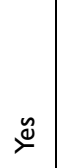 & 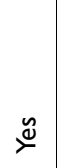 & & $\stackrel{\mathscr{\Perp}}{\tau}$ & $\stackrel{\stackrel{y}{*}}{x}$ & $\stackrel{\tilde{v}}{\check{\nu}}$ & $\stackrel{\mathscr{y}}{\tau}$ \\
\hline 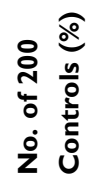 & & 0 & 0 & 0 & 0 & 0 & 0 & 0 & 0 & 0 & 0 & 0 & 0 & 0 & 0 & 0 & 0 & 0 & & 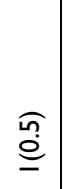 & 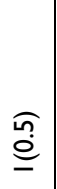 & 0 & 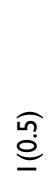 \\
\hline 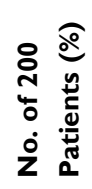 & & $\underbrace{\stackrel{n}{\leftrightarrows}}_{m}$ & 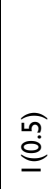 & 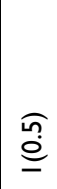 & 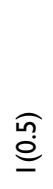 & 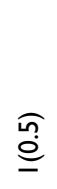 & $\underset{\sim}{\stackrel{\sigma}{\dot{~}}}$ & 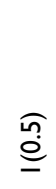 & $\begin{array}{l}\stackrel{\overbrace n}{\varrho} \\
\stackrel{\varrho}{\varrho}\end{array}$ & $\begin{array}{l}\sqrt[n]{e} \\
\stackrel{0}{0}\end{array}$ & 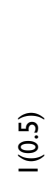 & 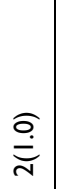 & $\begin{array}{l}\stackrel{\mathfrak{n}}{\varrho} \\
\stackrel{0}{0}\end{array}$ & $\begin{array}{l}\tilde{n} \\
\stackrel{\varrho}{=}\end{array}$ & 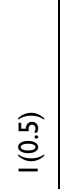 & 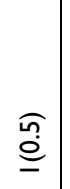 & 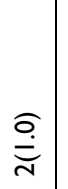 & $\underset{\sim}{\stackrel{\sigma}{\dot{N}}}$ & & $\stackrel{\widehat{n}}{\stackrel{\varrho}{\underline{e}}}$ & 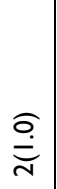 & $\begin{array}{l}\tilde{\mathfrak{n}} \\
\stackrel{\varrho}{\underline{e}}\end{array}$ & $\underset{\text { 오 }}{\dot{N}}$ \\
\hline 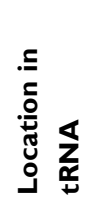 & & 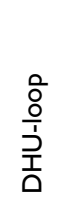 & $\begin{array}{l}\stackrel{O}{o} \\
\underline{o} \\
\underline{y} \\
F\end{array}$ & 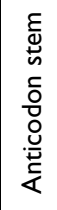 & 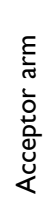 & 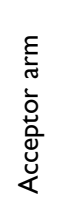 & 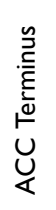 & 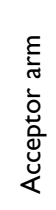 & $\begin{array}{l}\frac{0}{\circ} \\
\frac{\circ}{3} \\
\stackrel{1}{\Delta} \\
0\end{array}$ & $\begin{array}{l}\frac{0}{\circ} \\
\frac{0}{3} \\
\frac{1}{1} \\
0\end{array}$ & $\begin{array}{l}\frac{0}{\circ} \\
\frac{0}{1} \\
\stackrel{T}{1} \\
0\end{array}$ & $\begin{array}{l}\frac{0}{\circ} \\
\frac{\circ}{3} \\
\stackrel{1}{\Delta}\end{array}$ & 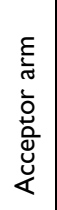 & 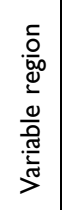 & 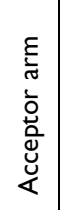 & $\begin{array}{l}\stackrel{0}{\circ} \\
\frac{0}{j} \\
\stackrel{2}{1} \\
\Delta\end{array}$ & 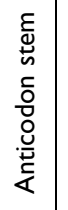 & 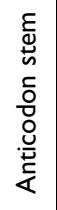 & & 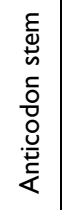 & 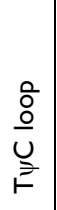 & $\begin{array}{l}\stackrel{0}{o} \\
\frac{o}{U} \\
⿱ 乛 \\
F\end{array}$ & 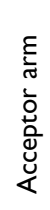 \\
\hline 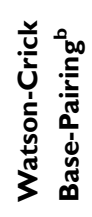 & & & & & $\begin{array}{l}\vec{u} \\
\dot{v}\end{array}$ & $\overrightarrow{P_{2}}$ & & $\overrightarrow{⿱ 乛 龰}$ & & $\overrightarrow{P_{j}}$ & $\vec{u}$ & $\begin{array}{l}\overleftarrow{U} \\
\dot{U}\end{array}$ & $\overrightarrow{P_{2}}$ & $\underset{5}{\overleftarrow{4}}$ & $\overrightarrow{P_{j}}$ & $\vec{u}$ & $\overrightarrow{P_{2}}$ & $\begin{array}{l}\vec{u} \\
\dot{v}\end{array}$ & & $\begin{array}{l}\vec{D} \\
\dot{<}\end{array}$ & & & ড্ড \\
\hline 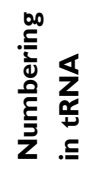 & & \pm & (요 & $\hat{m}$ & $\bar{r}$ & $m$ & $\Re$ & - & \pm & $=$ & $\underline{\alpha}$ & $\underline{m}$ & $R$ & $\stackrel{m}{q}$ & $\stackrel{\infty}{0}$ & 으 & बे & $\mathcal{F}$ & & ஓ & ก & in & 0 \\
\hline 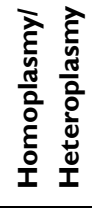 & & 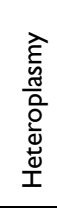 & 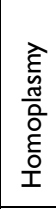 & 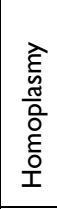 & 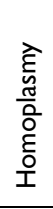 & 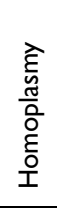 & 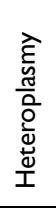 & 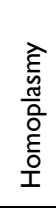 & 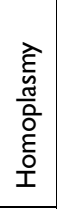 & 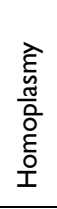 & 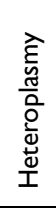 & 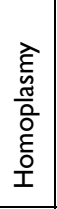 & 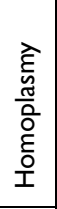 & $\begin{array}{l}\widehat{\hat{E}} \\
\frac{\tilde{g}}{0} \\
\overline{0} \\
\stackrel{0}{0} \\
\stackrel{0}{I} \\
\end{array}$ & 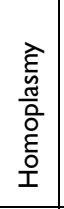 & 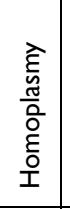 & 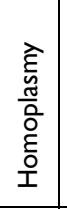 & 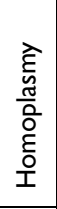 & & 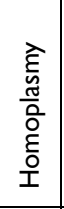 & 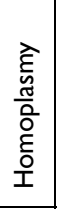 & 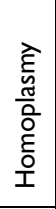 & 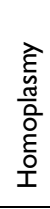 \\
\hline $\bar{u} \stackrel{\nwarrow}{\bigodot}$ & & 으 & œ & 으 & 으 & $\sigma$ & œ & œ & 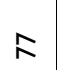 & \& & 으 & $\stackrel{\circ}{\circ}$ & ஃ & 으 & 으 & ळ & œ & $\stackrel{n}{n}$ & & 20 & $\approx$ & $\ddot{\sim}$ & m \\
\hline 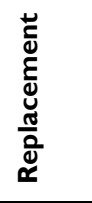 & 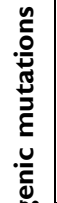 & $\begin{array}{l}0 \\
0 \\
8 \\
\varangle\end{array}$ & $\begin{array}{c}u \\
\stackrel{1}{*} \\
\vdash\end{array}$ & $\begin{array}{l}0 \\
0 \\
0 \\
\varangle\end{array}$ & $\begin{array}{l}\varangle \\
\stackrel{\leftrightarrow}{u}\end{array}$ & $\begin{array}{l}0 \\
\stackrel{8}{*} \\
\stackrel{<}{*}\end{array}$ & $\begin{array}{l}\cup \\
\stackrel{2}{\circ} \\
\vdash\end{array}$ & $\begin{array}{l}\cup \\
\stackrel{8}{*} \\
\stackrel{+}{<}\end{array}$ & $\stackrel{\vdash}{\stackrel{\leftrightarrow}{u}}$ & $\begin{array}{l}\cup \\
\stackrel{\leftrightarrow}{\circ} \\
\vdash\end{array}$ & $\begin{array}{l}\varangle \\
8 \\
ن\end{array}$ & 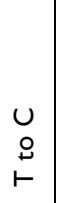 & $\begin{array}{l}\cup \\
\circ \\
\stackrel{\leftrightarrow}{<}\end{array}$ & $\begin{array}{l}0 \\
0 \\
8\end{array}$ & $\begin{array}{l}0 \\
0 \\
\dot{R}\end{array}$ & $\begin{array}{l}\varangle \\
\stackrel{8}{0} \\
0\end{array}$ & $\begin{array}{l}0 \\
0 \\
\&\end{array}$ & $\begin{array}{l}\varangle \\
8 \\
ن\end{array}$ & & $\begin{array}{l}0 \\
\dot{2} \\
\dot{<}\end{array}$ & $\stackrel{\vdash}{\circ}$ & $\begin{array}{l}0 \\
0 \\
\dot{0} \\
<\end{array}$ & $\begin{array}{l}\cup \\
\stackrel{8}{\leftarrow} \\
\vdash\end{array}$ \\
\hline 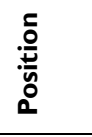 & 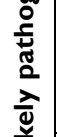 & 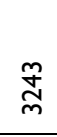 & ঐ্লি & 莓 & $\hat{f o j}$ & 苞 & 总 & 崅 & 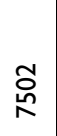 & 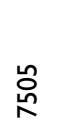 & $\frac{m}{\infty}$ & $\begin{array}{l}\tilde{o} \\
\text { o. } \\
\text { - }\end{array}$ & $\begin{array}{l}\text { 足 } \\
\text { O. } \\
\underline{0}\end{array}$ & 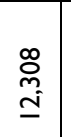 & 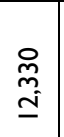 & 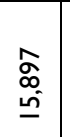 & 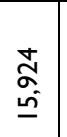 & $\begin{array}{l}\hat{\jmath} \\
\underline{\underline{\omega}}\end{array}$ & & :̊̊ & 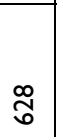 & $\tilde{\tilde{\omega}}$ & $\underline{\underline{0}}$ \\
\hline 心 & 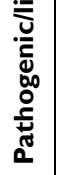 & 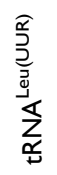 & & 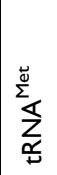 & & 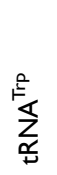 & 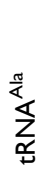 & & 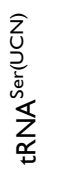 & & 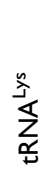 & 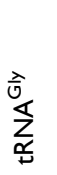 & & 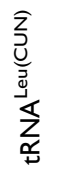 & & 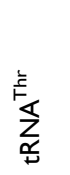 & & & 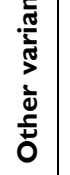 & $\underset{\substack{a \\
\frac{D}{a}}}{\stackrel{0}{a}}$ & & & 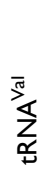 \\
\hline
\end{tabular}




\begin{tabular}{|c|c|c|c|c|c|c|c|c|c|c|c|c|c|c|c|c|c|c|c|c|}
\hline 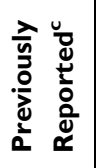 & $\stackrel{\mathscr{\nu}}{\check{\nu}}$ & $\stackrel{\tilde{\nu}}{\nu}$ & $\stackrel{\mathscr{\nu}}{\check{\nu}}$ & $\stackrel{\mathscr{y}}{\check{\nu}}$ & $\stackrel{\tilde{\nu}}{\nu}$ & $\stackrel{\tilde{\nu}}{\tau}$ & $\stackrel{y}{\check{\nu}}$ & $\stackrel{\mathscr{y}}{\tau}$ & $\stackrel{\mathscr{\nu}}{\check{\nu}}$ & 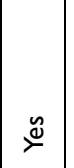 & 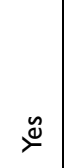 & $\stackrel{\tilde{\nu}}{\tau}$ & $\stackrel{\mathscr{y}}{\nu}$ & $\stackrel{\mathscr{y}}{\check{\nu}}$ & $\stackrel{\tilde{y}}{\nu}$ & $\stackrel{\mathscr{y}}{\check{\nu}}$ & $\stackrel{y}{\check{\nu}}$ & $\stackrel{\check{\nu}}{\check{\nu}}$ & $\stackrel{\mathscr{y}}{\check{\nu}}$ & $\stackrel{\tilde{y}}{\tau}$ \\
\hline 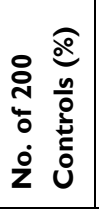 & 0 & $\stackrel{\widehat{o}}{\stackrel{\sim}{\sim}}$ & 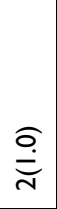 & 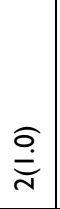 & 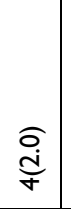 & 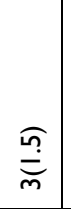 & $\begin{array}{l}\widetilde{n} \\
\stackrel{n}{n}\end{array}$ & 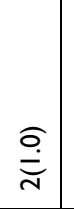 & 0 & 軼 & $\stackrel{\widehat{o}}{\stackrel{\sim}{\sim}}$ & 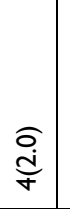 & 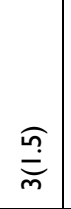 & $\underset{\stackrel{\sigma}{\mathfrak{g}}}{\widehat{f}}$ & 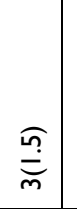 & $\begin{array}{l}\stackrel{\mathrm{O}}{\mathrm{q}} \\
\mathrm{g}\end{array}$ & $\begin{array}{l}\stackrel{\tilde{n}}{\varrho} \\
=\end{array}$ & 0 & 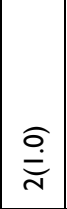 & $\stackrel{\widehat{O}}{\stackrel{\mathrm{J}}{\mathrm{T}}}$ \\
\hline 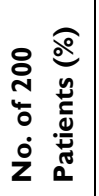 & 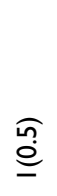 & 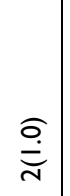 & 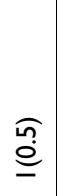 & 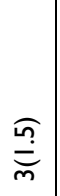 & 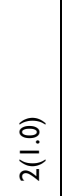 & 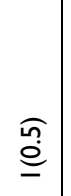 & $\begin{array}{l}\stackrel{\tilde{n}}{\varrho} \\
\stackrel{0}{0}\end{array}$ & 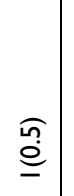 & $\widehat{\widehat{ָ}}$ & $\begin{array}{l}\sqrt[n]{n} \\
\stackrel{\varrho}{\varrho}\end{array}$ & $\begin{array}{c}\sqrt[\overbrace n]{0} \\
\stackrel{\varrho}{\varrho}\end{array}$ & $\underset{\sim}{\stackrel{\sigma}{\dot{*}}}$ & $\stackrel{\substack{n \\
\varrho}}{\varrho}$ & $\widehat{\bar{N}}$ & 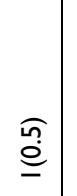 & $\widehat{\lesssim}$ & $\begin{array}{c}\stackrel{\tilde{n}}{\varrho} \\
\underline{\underline{e}}\end{array}$ & 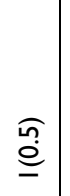 & $\begin{array}{c}\tilde{n} \\
\stackrel{\varrho}{\varrho} \\
0\end{array}$ & $\widehat{\bar{N}}$ \\
\hline 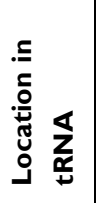 & 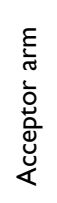 & 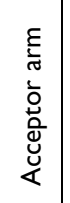 & 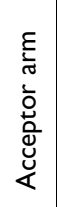 & $\begin{array}{l}\frac{o}{o} \\
\frac{o}{U} \\
\stackrel{z}{F}\end{array}$ & $\begin{array}{l}\stackrel{o}{o} \\
\frac{o}{U} \\
\stackrel{F}{F}\end{array}$ & $\begin{array}{l}\stackrel{o}{o} \\
\underline{o} \\
\stackrel{\Xi}{F} \\
F\end{array}$ & 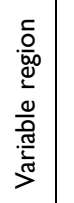 & $\begin{array}{l}0 \\
\frac{0}{1} \\
\frac{0}{3} \\
\text { 좀 }\end{array}$ & 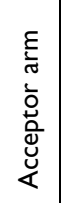 & 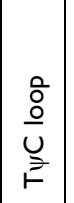 & $\begin{array}{l}\frac{0}{\circ} \\
\frac{0}{i} \\
\stackrel{1}{1} \\
\Delta\end{array}$ & $\begin{array}{l}\frac{o}{o} \\
\frac{o}{U} \\
⿱ 乛\end{array}$ & 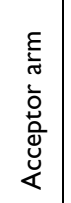 & $\begin{array}{l}\stackrel{o}{o} \\
\frac{o}{U} \\
\stackrel{F}{F}\end{array}$ & $\begin{array}{l}\frac{0}{0} \\
\frac{0}{j} \\
\stackrel{1}{0} \\
0\end{array}$ & 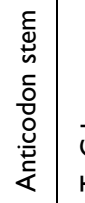 & $\begin{array}{l} \\
\stackrel{\circ}{o} \\
\frac{o}{U} \\
ٍ\end{array}$ & 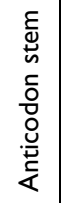 & 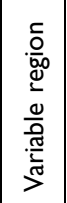 & $\begin{array}{l}\stackrel{\circ}{o} \\
\underline{\underline{O}} \\
\stackrel{F}{F}\end{array}$ \\
\hline 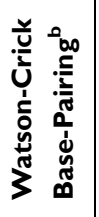 & $\underset{⿱ 亠 乂}{\stackrel{\leftarrow}{<}}$ & & 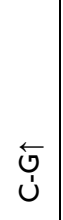 & & & & & & $\vec{u}$ & & & & & & & & & 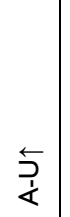 & & \\
\hline 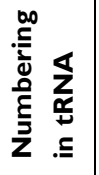 & $\hat{o}$ & $\stackrel{\infty}{m}$ & 0 & กิ & ๙ิ & $\overline{0}$ & बे & $\underline{0}$ & 0 & J & $\simeq$ & แे & 0 & பّ & $\underline{0}$ & $\stackrel{\infty}{\infty}$ & คำ & $\stackrel{\infty}{\sim}$ & $\bar{m}$ & 亡े \\
\hline 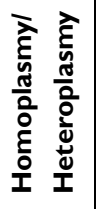 & 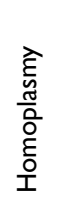 & 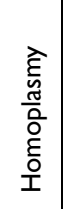 & 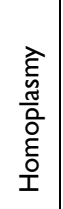 & 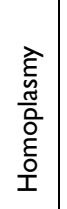 & 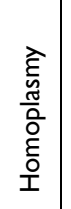 & 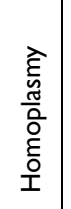 & 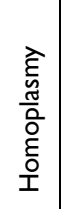 & 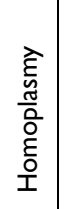 & 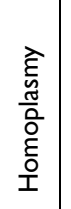 & 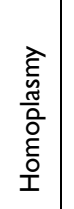 & 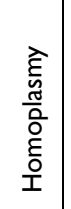 & 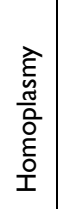 & 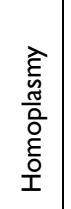 & 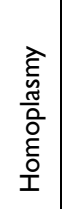 & 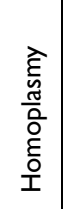 & 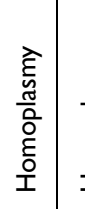 & 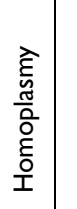 & 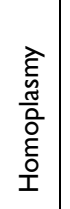 & 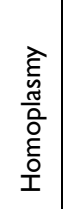 & 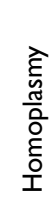 \\
\hline $\bar{u} \stackrel{\varpi}{\circlearrowleft}$ & $\bar{m}$ & $\stackrel{n}{\kappa}$ & $\infty$ & ติ & З & R & F & $\widehat{6}$ & $\infty$ & $\stackrel{8}{\circ}$ & $m$ & $\mathscr{r}$ & $=$ & o & 节 & 织 & $\stackrel{m}{\infty}$ & $\widehat{6}$ & 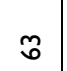 & ஃ \\
\hline 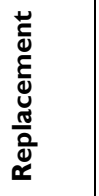 & 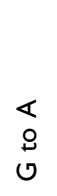 & $\begin{array}{l}u \\
\stackrel{2}{ } \\
\vdash\end{array}$ & $\begin{array}{c}u \\
\stackrel{2}{ } \\
\vdash\end{array}$ & 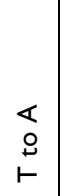 & $\stackrel{\leftarrow}{\stackrel{\leftrightarrow}{u}}$ & $\begin{array}{l}\varangle \\
\vdots \\
0\end{array}$ & $\begin{array}{l}0 \\
0 \\
\stackrel{8}{<}\end{array}$ & $\begin{array}{c}u \\
\stackrel{8}{ } \\
\vdash\end{array}$ & $\stackrel{\leftarrow}{\dot{s}}$ & $\begin{array}{l}u \\
\stackrel{2}{ } \\
\vdash\end{array}$ & $\begin{array}{r}- \\
\stackrel{\Delta}{u}\end{array}$ & $\begin{array}{l}0 \\
\dot{8} \\
\dot{<}\end{array}$ & $\begin{array}{c}u \\
\stackrel{2}{ } \\
\vdash\end{array}$ & $\begin{array}{c}\cup \\
\stackrel{8}{ } \\
\vdash\end{array}$ & $\stackrel{\vdash}{\stackrel{\circ}{u}}$ & \begin{tabular}{l|l}
0 & 1 \\
$\dot{0}$ & \\
$\stackrel{<}{*}$ & 1
\end{tabular} & \begin{tabular}{l|l}
$u$ & \\
$\stackrel{2}{*}$ & \\
$\vdash$
\end{tabular} & $\begin{array}{l}0 \\
0 \\
\stackrel{+}{<}\end{array}$ & $\begin{array}{r}\vdash \\
\stackrel{\leftrightarrow}{u}\end{array}$ & $\begin{array}{l}0 \\
0 \\
\&\end{array}$ \\
\hline 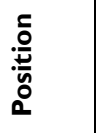 & 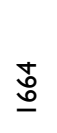 & $\underset{\stackrel{\aleph}{\sigma}}{\stackrel{\sigma}{\sigma}}$ & 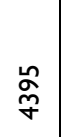 & 菒 & $\bar{\circ}$ & 䀡 & 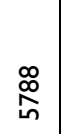 & $\overline{\bar{\infty}}$ & 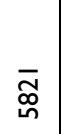 & $\overline{\hat{n}}$ & 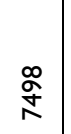 & $\underset{\infty}{\stackrel{m}{\tilde{~}}}$ & $\begin{array}{l}0 \\
\bar{t} \\
0\end{array}$ & 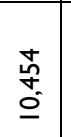 & $\frac{m}{\underline{n}}$ & $\begin{array}{l}\underline{\underline{N}} \\
\underline{\mathrm{N}}\end{array}$ & 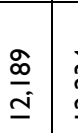 & $\begin{array}{l}\underset{N}{ \pm} \\
\text { I }\end{array}$ & 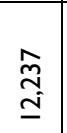 & 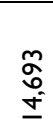 \\
\hline$\stackrel{\Xi}{\Phi}$ & & 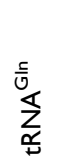 & & 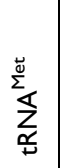 & 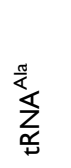 & & 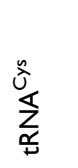 & & & 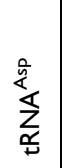 & 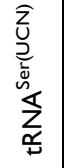 & 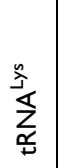 & 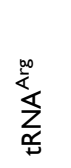 & & 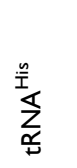 & & & 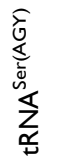 & & 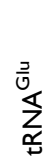 \\
\hline
\end{tabular}




\begin{tabular}{|c|c|c|c|c|c|c|c|}
\hline$\stackrel{\check{y}}{\check{\nu}}$ & $\stackrel{\Xi}{\check{\nu}}$ & $\stackrel{\tilde{\nu}}{\nu}$ & $\stackrel{\varpi}{\nu}$ & $\stackrel{\varpi}{\nu}$ & $\stackrel{y}{\nu}$ & $\stackrel{\Xi}{\check{\nu}}$ & $\stackrel{\tilde{y}}{\nu}$ \\
\hline$\underset{\mathrm{d}}{\stackrel{\mathrm{J}}{ }}$ & 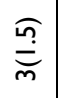 & 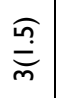 & 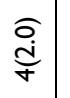 & 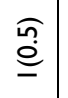 & 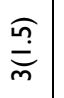 & 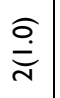 & 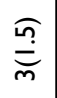 \\
\hline$\stackrel{\text { }}{\stackrel{\Omega}{\Theta}}$ & $\begin{array}{l}\stackrel{\mathfrak{n}}{\varrho} \\
\stackrel{0}{0}\end{array}$ & 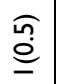 & 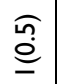 & $\begin{array}{l}\tilde{\tilde{n}} \\
\stackrel{0}{0}\end{array}$ & 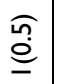 & $\begin{array}{c}\stackrel{\tilde{n}}{\varrho} \\
\stackrel{0}{0}\end{array}$ & $\begin{array}{l}\stackrel{\tilde{n}}{\varrho} \\
\stackrel{-}{0}\end{array}$ \\
\hline 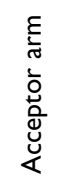 & $\begin{array}{l}\frac{0}{\circ} \\
\frac{\circ}{\grave{j}} \\
\stackrel{1}{\Delta}\end{array}$ & $\begin{array}{l}\frac{0}{\circ} \\
\frac{\circ}{j} \\
\frac{1}{\Delta} \\
\frac{1}{\Delta}\end{array}$ & $\begin{array}{l}\frac{0}{\circ} \\
\frac{\circ}{i} \\
\stackrel{1}{1} \\
口\end{array}$ & 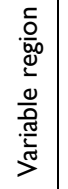 & $\begin{array}{l}\stackrel{o}{o} \\
\frac{o}{U} \\
\equiv \\
F\end{array}$ & 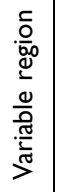 & 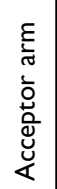 \\
\hline$\sim$ & $\underline{m}$ & $\simeq$ & 오 & $\stackrel{m}{q}$ & मे & $\underset{\sim}{ }$ & $n$ \\
\hline 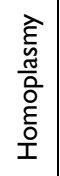 & 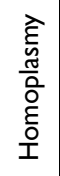 & 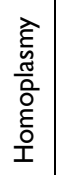 & 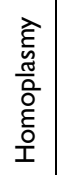 & 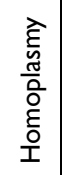 & 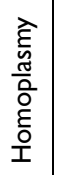 & 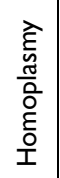 & 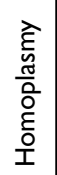 \\
\hline$\stackrel{\infty}{\circ}$ & 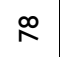 & $\widehat{\sigma}$ & น & $\bar{\alpha}$ & $\stackrel{\infty}{q}$ & $=$ & $\stackrel{\sim}{m}$ \\
\hline $\begin{array}{c}u \\
\stackrel{8}{ } \\
\vdash\end{array}$ & $\begin{array}{c}u \\
\stackrel{8}{ } \\
\vdash\end{array}$ & $\begin{array}{r}\vdash \\
\stackrel{\leftrightarrow}{u}\end{array}$ & $\begin{array}{l}\cup \\
\dot{0} \\
\dot{<}\end{array}$ & \begin{tabular}{l}
$\cup$ \\
O \\
\multirow{4}{*}{}
\end{tabular} & $\begin{array}{l}u \\
\& \\
⺊\end{array}$ & $\begin{array}{l}\vdash \\
\stackrel{8}{ } \\
ن\end{array}$ & $\begin{array}{l}\cup \\
\dot{0} \\
\stackrel{<}{<}\end{array}$ \\
\hline $\begin{array}{l}\stackrel{a}{\infty} \\
\stackrel{\infty}{\underline{0}}\end{array}$ & 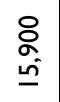 & 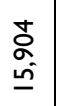 & $\begin{array}{l}\hat{a} \\
\underline{\underline{a}} \\
\underline{\hat{n}}\end{array}$ & $\begin{array}{l}\stackrel{\sigma}{\sigma} \\
\underline{\underline{n}}\end{array}$ & 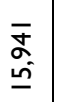 & $\begin{array}{l}8 \\
0 \\
\underline{0}\end{array}$ & $\begin{array}{l}\hat{\bar{o}} \\
\underline{0}\end{array}$ \\
\hline 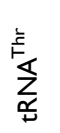 & & & & & & 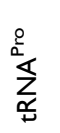 & \\
\hline
\end{tabular}

resided at DHU-loop, affected the conserved Watson-Crick base-pairings. Furthermore, variants $\mathrm{A} 4435 \mathrm{G}$ in tRNA ${ }^{\mathrm{Met}}$, A15924G and G15927A in tRNA ${ }^{\text {Thr }}$ occurred at anticodon stem may affect the steady-state level of mt-tRNAs. While variant A3290G in tRNA ${ }^{\text {Leu(UUR) }}$ occurred at T $\psi \mathrm{C}$ loop, variant $\mathrm{T} 5587 \mathrm{C}$ in tRNA ${ }^{\mathrm{Ala}}$ occurred at ACC Terminus, variant $\mathrm{A} 12330 \mathrm{G}$ in $\mathrm{tRNA}^{\mathrm{Leu(CUN)}}$ located at variable region may also affect the structure and function of mttRNAs.

\section{Whole Mitochondrial Genome Analysis of the 23 Subjects Carrying Putative Pathogenic Mt-tRNA Variants}

As shown in Table 2, 23 patients with T2DM carried the pathogenic/likely pathogenic mt-tRNA variants, accounting for $11.5 \%$ of the cases in our cohort. The age at onset of T2DM in these subjects ranged from 30 to 71 years. Moreover, a comprehensive medical history showed that 2 of 17 probands carrying putative pathogenic mt-tRNA variants had an obvious family history of T2DM, in particular, the family members of DM-101 and DM-102 carrying tRNA ${ }^{\text {Leu(UUR) }}$ A3243G and T3290C variants suffered both hearing loss and diabetes. There were variable clinical phenotypes of diabetes among these probands, with different levels of HbAlc, as well as FPG. In addition, analysis of entire mitochondrial genomes in 23 probands revealed 3 possible functional mtDNA variants: NADH dehydrogenase 1 (ND1) T3394C and T3398C, ND5 T12338C, which co-existed with tRNA ${ }^{\text {Leu(UUR) }}$ T3290C, tRNA $^{\text {Trp }}$ A5514G and tRNA ${ }^{\text {Gly }}$ T10003C, respectively. Interestingly, the T3394C (Tyr to His) and T3398C (Met to $\mathrm{Thr}$ ) variants occurred at very conserved nucleotides of ND1 polypeptide. ${ }^{33,34}$ While the ND5 T12338C (Met to $\mathrm{Thr}$ ) variant resulted in replacement of the first amino acid, translation-initiating methionine with a threonine, which may alter the respiratory function, as well as the processing of RNA precursors. ${ }^{35}$

The entire mtDNA sequences of 23 probands carrying these putative mt-tRNA variants were performed and then assigned to East Asian mitochondrial haplogroups based on the Phylotree database (http://www.phylotree.org/). ${ }^{36}$ As shown in Table 2, according to their distinct sets of polymorphisms, the mtDNA of 23 probands belonged to East Asian haplogroups F2, D4, G2b, N1a, N9a, G2a1, F1, D5b1b, C4c, A4, M11b, M11, D5a, U, D4b1, D4b2b, B5b and B5b1, respectively. ${ }^{29}$ 

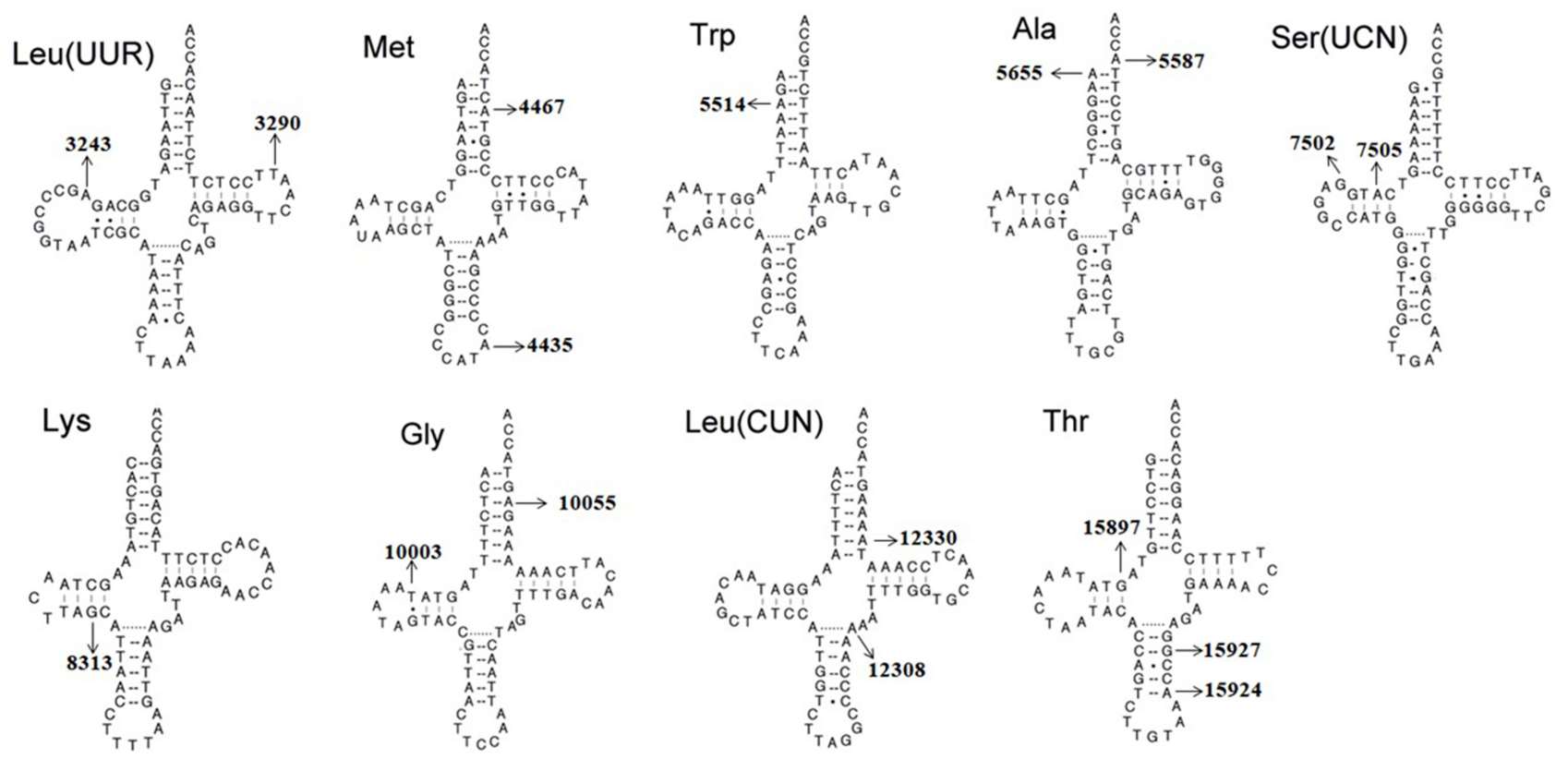

Figure 2 Summary of 17 pathogenic/likely pathogenic mt-tRNA variants at the cloverleaf structures of canonical tRNAs. Arrows indicate the positions of the variants in the mt-tRNA.

\section{Clinical and Biochemical Characterization of Two MIDD Pedigrees Carrying tRNA ${ }^{\text {Leu(UUR) }}$ Variants}

As shown in Figure 1, two Han Chinese families (DM-101 and DM-102) with MIDD were ascertained in Yantai Affiliated Hospital of Binzhou Medical University. A comprehensive history and physical examinations were performed to identify any clinical abnormalities, genetic factors related to diabetes in members of these two families. In DM-101 pedigree, the proband (III-5) was a 32-year-old woman who came from Yantai city of Shandong province. She began to suffer from T2DM when she was 30 (Table 3), she also developed bilateral hearing loss $(55 \mathrm{~dB}$ at right ear and $70 \mathrm{~dB}$ at left ear). Moreover, the family history suggested that other matrilineal relatives (II-3 and II-6) suffered from T2DM at different ages at onset. In particular, subject II-3 had profound hearing loss $(90 \mathrm{~dB}$ at right ear and $95 \mathrm{~dB}$ at left ear) and hypertension (145/80 $\mathrm{mmHg})$. Subject II-6 had moderate hearing impairment $(55 \mathrm{~dB}$ at right ear and $35 \mathrm{~dB}$ at left ear) and hypertension $(150 / 100 \mathrm{mmHg})$, whereas other members of this family were normal.

In DM-102 pedigree, the proband (II-10) was a 68 -year -old woman who also lived in Yantai city of Shandong province. She suffered from T2DM when she was 55 . Comprehensive physical examinations indicated that she had very high BP (140/95 $\mathrm{mmHg}$ ) for 3 years, she also exhibited mild hearing impairment ( $38 \mathrm{~dB}$ at right ear and $40 \mathrm{~dB}$ at left ear). Genetic counseling revealed that matrilineal relatives (II-5, II-8, III-7 and III-3) were also T2DM carriers. Besides, other members in DM-102 were normal subjects. The clinical and biochemical data of these subjects are listed in Table 3.

\section{Analysis of mtDNA Variants}

The maternally inherited pattern of these two pedigrees indicated that mitochondrial dysfunctions may be involved in the pathogenesis of MIDD. In order to investigate the contributions of mtDNA variants to MIDD, the complete mitochondrial genomes of matrilineal relatives from these families (DM-101: II-3, II-6 and III-5; DM102: II-5, II-8, II-10 and III-7) were PCR amplified and sequenced by ABI 3730 automated DNA instrument, subsequently the data was compared with the rCRS and the mtDNA variants were screened. As can be seen in Table 4, matrilineal relatives of these pedigrees harbored 76 mtDNA variants, of these, 21 variants occurred at D-loop, 3 variants in $12 \mathrm{~S}$ rRNA, 3 variants in $16 \mathrm{~S}$

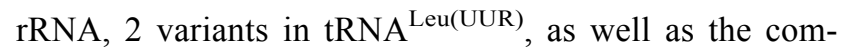
mon COII/tRNA ${ }^{\text {Lys }}$ intergenic 9-bp deletion occurred at position between 8271 and 8279 . Other variants were localized at oxidative phosphorylation (OXPHOS)related genes. In addition, 12 missense variants were 
Table 2 Mitochondrial Genetic Background and Clinical Features of 23 Probands Carrying One of the Pathogenic/Likely Pathogenic T2DM-Associated tRNA Variants

\begin{tabular}{|c|c|c|c|c|c|c|c|c|c|c|}
\hline Genes & Variants & Probands & Gender & $\begin{array}{l}\text { Age } \\
\text { at } \\
\text { Test } \\
\text { (Year) }\end{array}$ & $\begin{array}{l}\text { Age at } \\
\text { Onset } \\
\text { (Year) }\end{array}$ & $\begin{array}{l}\text { HbAlc } \\
(\%)\end{array}$ & $\begin{array}{l}\text { Glucose } \\
\text { (Oh) }\end{array}$ & $\begin{array}{l}\text { Family } \\
\text { History }\end{array}$ & $\begin{array}{l}\text { Other } \\
\text { Functional } \\
\text { mtDNA } \\
\text { Variants }\end{array}$ & Haplogroup \\
\hline \multirow[t]{4}{*}{ tRNA ${ }^{\text {Leu(UUR) }}$} & A3243G & DM-I0I & $M$ & 32 & 30 & 7.1 & 9.1 & Yes & l & F2 \\
\hline & & DM-210 & $\mathrm{F}$ & 44 & 38 & 6.5 & 10.8 & No & l & F2 \\
\hline & & DM-225 & $\mathrm{F}$ & 51 & 42 & 7.0 & II.I & No & I & $\mathrm{F} 2$ \\
\hline & T3290C & DM-I02 & M & 68 & 55 & 6.9 & 5.6 & Yes & NDI T3394C & D4 \\
\hline \multirow[t]{2}{*}{$\mathrm{tRNA}^{\mathrm{Met}}$} & A4435G & DM-132 & $M$ & 70 & 65 & 7.1 & 9.1 & No & l & $\mathrm{G} 2 \mathrm{~b}$ \\
\hline & C4467A & $D M-126$ & $M$ & 52 & 41 & 7.2 & 4.8 & No & l & $\mathrm{Nla}$ \\
\hline tRNA $A^{\text {Trp }}$ & A55I4G & DM-108 & $\mathrm{F}$ & 46 & 39 & 7.9 & 8.7 & No & NDI T3398C & $\mathrm{N} 9 \mathrm{a}$ \\
\hline \multirow[t]{3}{*}{ tRNA ${ }^{\text {Ala }}$} & \multirow[t]{2}{*}{ T5587C } & DM-2I3 & $\mathrm{F}$ & 66 & 61 & 8.3 & 5.2 & No & 1 & G2al \\
\hline & & DM-259 & $M$ & 59 & 52 & 8.0 & 5.6 & No & l & $\mathrm{FI}$ \\
\hline & A5655G & DM-277 & $M$ & 41 & 38 & 7.7 & 7.0 & No & l & D5blb \\
\hline \multirow[t]{2}{*}{$\operatorname{tRNA}^{\mathrm{Ser}(\mathrm{UCN})}$} & C7502T & DM-239 & M & 39 & 33 & 7.4 & 4.9 & No & I & $\mathrm{C} 4 \mathrm{c}$ \\
\hline & T7505C & DM-184 & $\mathrm{F}$ & 57 & 53 & 7.0 & 13.0 & No & l & $\mathrm{FI}$ \\
\hline tRNA $A^{\text {Lys }}$ & G83I3A & DM-199 & $\mathrm{F}$ & 65 & 50 & 6.6 & 11.6 & No & 1 & $\mathrm{~A} 4$ \\
\hline \multirow[t]{3}{*}{ tRNA $^{\text {Gly }}$} & \multirow[t]{2}{*}{ TI0003C } & DM-217 & $\mathrm{F}$ & 71 & 70 & 6.8 & 8.0 & No & ND5 TI2338C & MIIb \\
\hline & & DM-290 & $M$ & 66 & 61 & 6.9 & 7.9 & No & l & MII \\
\hline & Al0055G & DM-255 & $M$ & 49 & 41 & 7.2 & 5.5 & No & I & D5a \\
\hline \multirow[t]{2}{*}{ tRNA ${ }^{\operatorname{Leu}(C U N)}$} & Al2308G & DM-230 & $M$ & 46 & 39 & 7.9 & 5.8 & No & l & $U$ \\
\hline & AI2330G & DM-222 & $M$ & 55 & 51 & 8.0 & 6.0 & No & 1 & $\mathrm{~F} 2$ \\
\hline \multirow[t]{5}{*}{$\mathrm{tRNA}^{\mathrm{Thr}}$} & GI5897A & DM-266 & M & 57 & 53 & 7.4 & 5.1 & No & l & D4b I \\
\hline & \multirow[t]{2}{*}{ AI5924G } & DM-277 & $\mathrm{F}$ & 79 & 71 & 7.3 & 6.8 & No & 1 & MII \\
\hline & & DM-28I & $\mathrm{F}$ & 60 & 58 & 7.1 & 6.6 & No & l & $\mathrm{D} 4 \mathrm{~b} 2 \mathrm{~b}$ \\
\hline & \multirow[t]{2}{*}{ GI5927A } & DM-155 & M & 59 & 52 & 6.8 & 6.8 & No & I & B5b \\
\hline & & DM-260 & $\mathrm{F}$ & 61 & 60 & 6.6 & 7.0 & No & l & B5bl \\
\hline
\end{tabular}

Abbreviations: T2DM, type 2 diabetes mellitus; mtDNA, mitochondrial DNA; M, male; F, female.

identified, including ND1 T3394C (Tyr to His), ND2 T5442C (Phe to Leu), CO2 G7598A (Ala to Thr), ATP6 (A6) A8701G (Thr to Ala) and A8860G (Thr to Ala), ND3 A10398G (Thr to Ala), ND4L T10609C (Met to Thr) and A10750G (Asn to Ser), ND5 G12406A (Val to Ile) and G13928C (Ser to Thr), CytB C14766T (Thr to Ile) and A15326G (Thr to Ala). To further assess their pathogenicity, phylogenetic approach was carried out to see the evolutionary conservation of each variant, especially in mouse, ${ }^{37}$ bovine $^{38}$ and Xenopus laevis. ${ }^{39} \mathrm{We}$ found that except for the $\mathrm{A} 3243 \mathrm{G}$ and $\mathrm{T} 3290 \mathrm{C}$ in
tRNA ${ }^{\text {Leu(UUR) }}$, the $\mathrm{T} 3394 \mathrm{C}$ in ND1, other variants showed lower levels of CIs (Figure 3 and 4). Moreover, the $\mathrm{A} 3243 \mathrm{G}$ and $\mathrm{T} 3290 \mathrm{C}$ variants were not detected in 200 controls, suggesting that they may be involved in the pathogenesis of MIDD.

\section{Mutational Analysis of GJB2 Gene}

Mutations in $G J B 2$ were the important causes for hearing loss, ${ }^{40}$ to examine the contributions of $G J B 2$ to deafness expression, we conducted a mutational screening for $G J B 2$ gene in matrilineal relatives of these MIDD pedigrees 
Table 3 Summary of Clinical and Biochemical Data for Several Members in These Two Families with Maternally Inherited Diabetes and Deafness

\begin{tabular}{|c|c|c|c|c|c|c|c|c|c|}
\hline Subjects & Gender & $\begin{array}{l}\text { Age at } \\
\text { Test } \\
\text { (Years) }\end{array}$ & $\begin{array}{l}\text { Age at } \\
\text { Onset } \\
\text { (Years) }\end{array}$ & $\begin{array}{l}\text { HbAlc } \\
\text { (\%) }\end{array}$ & $\begin{array}{l}\text { Glucose } \\
\text { (oh) } \\
(\mathrm{mmol} / \mathrm{L})\end{array}$ & $\begin{array}{l}\text { Glucose } \\
(2 \mathrm{~h}) \\
(\mathrm{mmol} / \mathrm{L})\end{array}$ & $\begin{array}{l}\text { BP } \\
(\mathrm{mmHg})\end{array}$ & $\begin{array}{l}\text { PTA (dB) } \\
\text { Right/Left } \\
\text { Ear }\end{array}$ & $\begin{array}{l}\text { Level of } \\
\text { Hearing } \\
\text { Loss }\end{array}$ \\
\hline DM-I0I (II-3) & Male & 55 & 50 & 6.6 & 7.7 & 14.2 & $145 / 80$ & $90 / 95$ & Profound \\
\hline DM-I0I (II-6) & Female & 58 & 45 & 7.0 & 8.0 & 12.6 & $150 / 100$ & $55 / 35$ & Moderate \\
\hline DM-I0I (III-5) & Female & 32 & 30 & 7.1 & 9.1 & 14.8 & $130 / 75$ & $55 / 70$ & Severe \\
\hline DM-I02 (II-5) & Male & 65 & 50 & 6.8 & 5.1 & 7.8 & $145 / 95$ & $20 / 20$ & Normal \\
\hline DM-I02 (II-8) & Female & 60 & 49 & 7.3 & 8.2 & 14.6 & $135 / 80$ & $23 / 17$ & Normal \\
\hline DM-I02 (II-I0) & Female & 68 & 55 & 6.9 & 5.6 & 8.0 & $140 / 95$ & $38 / 40$ & Mild \\
\hline DM-I02 (III-7) & Female & 40 & 38 & 6.5 & 5.2 & 7.1 & $130 / 85$ & $52 / 45$ & Moderate \\
\hline DM-I02 (III-3) & Female & 36 & I & 5.6 & 4.8 & 6.9 & $125 / 75$ & $20 / 15$ & Normal \\
\hline
\end{tabular}

Abbreviations: HbAlc, glycosylated hemoglobin; BP, blood pressure; PTA, pure-tone audiometry; dB, decibel.

(DM-101: II-3, II-6 and III-5; DM-102: II-5, II-8, II-10 and III-7). However, we failed to detect any variants in GJB2 gene, suggesting that GJB2 may not play an active role in the phenotypic manifestation of MIDD in these families.

\section{Mutational Screening of TRMU Gene}

Previous study showed that variants in TRMU may modulate the clinical manifestation of deafness-associated mitochondrial A1555G or C1494T mutation. ${ }^{41}$ To examine whether TRMU played an important role in MIDD expression, we screened the TRMU A10S variant in matrilineal relatives of these families. But we did not detect the presence of A10S variant suggested that TRMU may not be involved in the pathogenesis of MIDD in these families.

\section{Discussion}

In the present case-control study, we analyzed the frequencies of mt-tRNA variants in 200 patients with T2DM and 200 controls. Through PCR and direct sequence analysis, a total of 49 genetic variants in mt-tRNA genes were identified. By focusing only on variants that were evolutionary conserved, presented $<1 \%$ in controls and predicted to induce functional or structural changes in mt-tRNA molecules, we were able to identify 17 pathogenic/likely pathogenic mt-tRNA variants in 23 probands with T2DM. Among them, the heteroplasmic A3243G in tRNA ${ }^{\text {Leu(UUR) }}$ was one of the most common T2DM-associated pathogenic mutations. ${ }^{42}$ This mutation also led to mitochondrial encephalomyopathy, lactic acidosis and stroke-like symptoms (MELAS). ${ }^{43}$ Molecular analysis revealed that this mutation reduced the steady-state level, aminoacylation, as well as codon recognition of tRNA ${ }^{\mathrm{Leu}(\mathrm{UUR})}{ }^{44}$ As a result, the A3243G mutation caused the pre-termination of transcription and expression impeding of normal rRNA, thus compromising mitochondrial protein synthesis, ATP synthesis and organic metabolism. ${ }^{45}$ While the homoplasmic T3290C variant occurred at position 59 in the $\mathrm{T} \psi \mathrm{C}$ loop of tRNA $^{\text {Leu(UUR) }}$, which had been regarded as a risk factor for hypertension. ${ }^{46}$ In addition, the A4435G variant was localized at immediately $3^{\prime}$ end to the anticodon, corresponding to the conventional position 37 of tRNA ${ }^{\text {Met }} 47$ The adenine (A37) at this position of tRNA ${ }^{\text {Met }}$ was extremely conserved from bacteria to human mitochondria. ${ }^{48}$ Furthermore, the $\mathrm{A} 4435 \mathrm{G}$ variant introduced an $\mathrm{m}^{1} \mathrm{G} 37$ modification of tRNA ${ }^{\text {Met }}$, altered its structure and function. Functional analysis of cybrid cells harboring this variant revealed a markedly diminished ATP levels and mitochondrial membrane potential (MMP), and increased reactive oxygen species (ROS) production. ${ }^{48}$ While the C4467A variant occurred at $3^{\prime}$ end of tRNA ${ }^{\mathrm{Met}}$, which was the processing site for the tRNA ${ }^{\text {Met }} 3^{\prime}$ end precursors of the light strand and was predicted to alter A-U base pairing (A1-U72) at the aminoacyl acceptor stem of tRNA ${ }^{\text {Met }} 49$ The C4467A variant may impair the cleavage of polycistronic mtRNA transcripts into mature mt-tRNA species at the tRNAase $\mathrm{Z}$ cleavage site. ${ }^{50}$ Thus, the point variant located at the $3^{\prime}$ end was important for both mt-tRNA synthesis and function. ${ }^{51}$ Moreover, the homoplasmic A5514G variant disrupted an A-U base-pair within the acceptor stem of tRNA ${ }^{\text {Trp }}$, had been reported to be associated with mitochondria encephalomyopathy. ${ }^{52}$ Interestingly, two variants (A5655G and T5587C) were identified in $\mathrm{tRNA}^{\mathrm{Ala}}$ gene, in fact, $\mathrm{A} 5655 \mathrm{G}$ variant was 
Table 4 mtDNA Sequence Variants in Two Chinese Families with Maternally Inherited Diabetes and Deafness

\begin{tabular}{|c|c|c|c|c|c|c|c|}
\hline Gene & Position & Alternation & $\begin{array}{l}\text { Conservation (H/B/M/ } \\
\text { X) }\end{array}$ & rCRS $^{b}$ & $\begin{array}{l}\text { DM- } \\
101\end{array}$ & $\begin{array}{l}\text { DM- } \\
102\end{array}$ & $\begin{array}{l}\text { Previously } \\
\text { Reported }^{c}\end{array}$ \\
\hline D-loop & $\begin{array}{l}73 \\
143 \\
146 \\
150 \\
195 \\
207 \\
263 \\
310 \\
374 \\
489 \\
514 \\
515 \\
523 \\
16,051 \\
16,093 \\
16,129 \\
16,175 \\
16,189 \\
16,223 \\
16,311 \\
16,526\end{array}$ & $\begin{array}{l}\text { A to } G \\
G \text { to } A \\
T \text { to } C \\
C \text { to } T \\
T \text { to } C \\
G \text { to } A \\
A \text { to } G \\
T \text { to } T C / C T C \\
A \text { to } G \\
T \text { to } C \\
\text { DelC } \\
\text { DelA } \\
\text { Del } A \\
A \text { to } G \\
T \text { to } C \\
G \text { to } A \\
A \text { to } T \\
T \text { to } C \\
C \text { to } T \\
T \text { to } C \\
G \text { to } A\end{array}$ & & $\begin{array}{l}\text { A } \\
\text { G } \\
T \\
C \\
T \\
G \\
A \\
T \\
A \\
T \\
C \\
A \\
A \\
A \\
T \\
G \\
A \\
T \\
C \\
T \\
\text { G }\end{array}$ & $\begin{array}{l}\text { G } \\
\text { T } \\
\text { A } \\
\text { TC } \\
\text { G } \\
\text { C } \\
\text { DelC } \\
\text { Del A } \\
\text { G } \\
\text { C } \\
\text { A } \\
\text { C } \\
\text { T } \\
\text { C } \\
\text { A }\end{array}$ & $\begin{array}{l}\text { G } \\
\text { C } \\
\text { C } \\
\text { G } \\
\text { CTC } \\
\text { C } \\
\text { DelA } \\
\text { G } \\
\text { A } \\
\text { T } \\
\text { C } \\
\text { T } \\
\text { C }\end{array}$ & $\begin{array}{l}\text { Yes } \\
\text { Yes } \\
\text { Yes } \\
\text { Yes } \\
\text { Yes } \\
\text { Yes } \\
\text { Yes } \\
\text { Yes } \\
\text { Yes } \\
\text { Yes } \\
\text { Yes } \\
\text { Yes } \\
\text { Yes } \\
\text { Yes } \\
\text { Yes } \\
\text { Yes } \\
\text { Yes } \\
\text { Yes } \\
\text { Yes } \\
\text { Yes } \\
\text { Yes }\end{array}$ \\
\hline I $2 \mathrm{~S}$ rRNA & $\begin{array}{l}750 \\
1041 \\
1438\end{array}$ & $\begin{array}{l}A \text { to } G \\
A \text { to } G \\
A \text { to } G\end{array}$ & $\begin{array}{l}\text { A/G/G/- } \\
\text { A/T/T/T } \\
\text { A/A/A/G }\end{array}$ & $\begin{array}{l}A \\
A \\
A\end{array}$ & $\begin{array}{l}\text { G } \\
\text { G }\end{array}$ & $\begin{array}{l}\mathrm{G} \\
\mathrm{G} \\
\mathrm{G}\end{array}$ & $\begin{array}{l}\text { Yes } \\
\text { Yes } \\
\text { Yes }\end{array}$ \\
\hline I6S rRNA & $\begin{array}{l}2706 \\
3010 \\
3107\end{array}$ & $\begin{array}{l}A \text { to } G \\
G \text { to } A \\
\text { Del } N\end{array}$ & $\begin{array}{l}\mathrm{A} / \mathrm{G} / \mathrm{A} / \mathrm{A} \\
\mathrm{G} / \mathrm{G} / \mathrm{A} / \mathrm{A}\end{array}$ & $\begin{array}{l}\mathrm{A} \\
\mathrm{G} \\
\mathrm{N}\end{array}$ & $\begin{array}{l}\text { G } \\
\text { Del N }\end{array}$ & $\begin{array}{l}\text { G } \\
\text { A } \\
\text { Del } N\end{array}$ & $\begin{array}{l}\text { Yes } \\
\text { Yes } \\
\text { Yes }\end{array}$ \\
\hline tRNA ${ }^{\text {Leu(UUR) }}$ & $\begin{array}{l}3243 \\
3290\end{array}$ & $\begin{array}{l}\text { A to } G \\
T \text { to } C\end{array}$ & $\begin{array}{l}\mathrm{A} / \mathrm{A} / \mathrm{A} / \mathrm{A} \\
\mathrm{T} / \mathrm{T} / \mathrm{T} / \mathrm{T}\end{array}$ & $\begin{array}{l}\mathrm{A} \\
\mathrm{T}\end{array}$ & G & C & $\begin{array}{l}\text { Yes } \\
\text { Yes }\end{array}$ \\
\hline NDI & $\begin{array}{l}3394 \\
3483 \\
3970 \\
4071\end{array}$ & $\begin{array}{l}T \text { to } C \text { (Tyr to His) } \\
G \text { to } A \\
C \text { to } T \\
C \text { to } T\end{array}$ & $Y / Y / Y / Y$ & $\begin{array}{l}\mathrm{T} \\
\mathrm{G} \\
\mathrm{C} \\
\mathrm{C}\end{array}$ & $\begin{array}{l}\mathrm{A} \\
\mathrm{T}\end{array}$ & $\begin{array}{l}\mathrm{C} \\
\mathrm{T}\end{array}$ & $\begin{array}{l}\text { Yes } \\
\text { Yes } \\
\text { Yes } \\
\text { Yes }\end{array}$ \\
\hline ND2 & $\begin{array}{l}4769 \\
4850 \\
5442\end{array}$ & $\begin{array}{l}\text { A to } G \\
C \text { to } T \\
T \text { to } C \text { (Phe to Leu) }\end{array}$ & F/F/M/L & $\begin{array}{l}\mathrm{A} \\
\mathrm{C} \\
\mathrm{T}\end{array}$ & $\begin{array}{l}\mathrm{G} \\
\mathrm{T}\end{array}$ & $\begin{array}{l}\mathrm{G} \\
\mathrm{C}\end{array}$ & $\begin{array}{l}\text { Yes } \\
\text { Yes } \\
\text { Yes }\end{array}$ \\
\hline $\mathrm{COI}$ & $\begin{array}{l}6392 \\
6455 \\
6599 \\
6962 \\
7028 \\
7250 \\
7337\end{array}$ & $\begin{array}{l}T \text { to } C \\
C \text { to } T \\
A \text { to } G \\
G \text { to } A \\
C \text { to } T \\
A \text { to } G \\
G \text { to } A\end{array}$ & & $\begin{array}{l}T \\
C \\
A \\
G \\
C \\
A \\
G\end{array}$ & $\begin{array}{l}\text { C } \\
\\
\text { G } \\
\text { A }\end{array}$ & $\begin{array}{l}\mathrm{C} \\
\mathrm{T} \\
\mathrm{G} \\
\mathrm{A} \\
\mathrm{T} \\
\mathrm{G} \\
\mathrm{A}\end{array}$ & $\begin{array}{l}\text { Yes } \\
\text { Yes } \\
\text { Yes } \\
\text { Yes } \\
\text { Yes } \\
\text { Yes } \\
\text { Yes }\end{array}$ \\
\hline $\mathrm{CO} 2$ & $\begin{array}{l}7598 \\
7805\end{array}$ & $\begin{array}{l}\text { G to } A \text { (Ala to Thr) } \\
G \text { to } A\end{array}$ & $\mathrm{~A} / \mathrm{M} / \mathrm{F} / \mathrm{S}$ & $\begin{array}{l}\mathrm{G} \\
\mathrm{G}\end{array}$ & & $\begin{array}{l}A \\
A\end{array}$ & $\begin{array}{l}\text { Yes } \\
\text { Yes }\end{array}$ \\
\hline NC7 & $828 I-8289$ & Del 9-bp & & СССССТСТА & & Del 9-bp & Yes \\
\hline
\end{tabular}


Table 4 (Continued).

\begin{tabular}{|c|c|c|c|c|c|c|c|}
\hline Gene & Position & Alternation & $\begin{array}{l}\text { Conservation }(\mathrm{H} / \mathrm{B} / \mathrm{M} / \\
\mathrm{X})^{\mathrm{a}}\end{array}$ & rCRS $^{b}$ & $\begin{array}{l}\text { DM- } \\
101\end{array}$ & $\begin{array}{l}\text { DM- } \\
102\end{array}$ & $\begin{array}{l}\text { Previously } \\
\text { Reported }^{c}\end{array}$ \\
\hline A8 & 8440 & A to $G$ & & A & G & & Yes \\
\hline A6 & $\begin{array}{l}8701 \\
8860\end{array}$ & $\begin{array}{l}\text { A to } G \text { (Thr to Ala) } \\
\text { A to } G \text { (Thr to Ala) }\end{array}$ & $\begin{array}{l}\mathrm{T} / \mathrm{S} / \mathrm{L} / \mathrm{Q} \\
\mathrm{T} / \mathrm{A} / \mathrm{A} / \mathrm{T}\end{array}$ & $\begin{array}{l}A \\
A\end{array}$ & G & $\begin{array}{l}\mathrm{G} \\
\mathrm{G}\end{array}$ & $\begin{array}{l}\text { Yes } \\
\text { Yes }\end{array}$ \\
\hline $\mathrm{CO} 3$ & $\begin{array}{l}9540 \\
9824 \\
9896\end{array}$ & $\begin{array}{l}T \text { to } C \\
T \text { to } C \\
\text { A to } G\end{array}$ & & $\begin{array}{l}\mathrm{T} \\
\mathrm{T} \\
\mathrm{A}\end{array}$ & $\begin{array}{l}\mathrm{C} \\
\mathrm{C}\end{array}$ & $\begin{array}{l}\text { C } \\
\text { G }\end{array}$ & $\begin{array}{l}\text { Yes } \\
\text { Yes } \\
\text { Yes }\end{array}$ \\
\hline ND3 & $\begin{array}{l}10,310 \\
10,398 \\
10,400\end{array}$ & $\begin{array}{l}G \text { to } A \\
A \text { to } G \text { (Thr to Ala) } \\
C \text { to } T\end{array}$ & $\mathrm{~T} / \mathrm{T} / \mathrm{T} / \mathrm{A}$ & $\begin{array}{l}\mathrm{G} \\
\mathrm{A} \\
\mathrm{C}\end{array}$ & $\begin{array}{l}\mathrm{G} \\
\mathrm{T}\end{array}$ & A & $\begin{array}{l}\text { Yes } \\
\text { Yes } \\
\text { Yes }\end{array}$ \\
\hline ND4L & $\begin{array}{l}10609 \\
10,750\end{array}$ & $\begin{array}{l}T \text { to } C \text { (Met to Thr) } \\
\text { A to } G \text { (Asn to Ser) }\end{array}$ & $\begin{array}{l}M / T / T / T \\
N / N / N / N\end{array}$ & $\begin{array}{l}\mathrm{T} \\
\mathrm{A}\end{array}$ & C & G & $\begin{array}{l}\text { Yes } \\
\text { Yes }\end{array}$ \\
\hline ND4 & $\begin{array}{l}10,683 \\
10,873 \\
11,719 \\
11,926\end{array}$ & $\begin{array}{l}G \text { to } A \\
T \text { to } C \\
G \text { to } A \\
A \text { to } C\end{array}$ & & $\begin{array}{c}\mathrm{G} \\
\mathrm{T} \\
\mathrm{G} \\
\mathrm{A}\end{array}$ & $\begin{array}{l}\text { A } \\
\text { C }\end{array}$ & $\begin{array}{l}\mathrm{C} \\
\mathrm{A}\end{array}$ & $\begin{array}{l}\text { Yes } \\
\text { Yes } \\
\text { Yes } \\
\text { Yes }\end{array}$ \\
\hline ND5 & $\begin{array}{l}12,360 \\
12,406 \\
12,705 \\
12,882 \\
13,152 \\
13,708 \\
13,759 \\
13,928\end{array}$ & $\begin{array}{l}\text { A to } G \\
G \text { to } A \text { (Val to lle) } \\
C \text { to } G \\
C \text { to } T \\
A \text { to } G \\
G \text { to } A \\
G \text { to } A \\
G \text { to } C \text { (Ser to Thr) }\end{array}$ & $\begin{array}{l}\text { V/F/S/F } \\
\text { S/T/S/T }\end{array}$ & $\begin{array}{l}\text { A } \\
\text { G } \\
C \\
C \\
A \\
G \\
G \\
G\end{array}$ & $\begin{array}{l}\mathrm{G} \\
\mathrm{G} \\
\mathrm{T} \\
\mathrm{A}\end{array}$ & $\begin{array}{l}A \\
G \\
T \\
G \\
\\
A \\
C\end{array}$ & $\begin{array}{l}\text { Yes } \\
\text { Yes } \\
\text { Yes } \\
\text { Yes } \\
\text { Yes } \\
\text { Yes } \\
\text { Yes } \\
\text { Yes }\end{array}$ \\
\hline ND6 & $|4,3| \mid$ & $\mathrm{T}$ to $\mathrm{C}$ & & $\mathrm{T}$ & & C & Yes \\
\hline$C y t B$ & $\begin{array}{l}14766 \\
14,783 \\
15,040 \\
15,043 \\
15,30 \mid \\
15,326\end{array}$ & $\begin{array}{l}C \text { to } T \text { (Thr to lle) } \\
T \text { to } C \\
C \text { to } T \\
G \text { to } A \\
G \text { to } A \\
\text { A to } G \text { (Thr to Ala) }\end{array}$ & $\begin{array}{l}\mathrm{T} / \mathrm{S} / \mathrm{I} / \mathrm{S} \\
\mathrm{T} / \mathrm{M} / \mathrm{I} / \mathrm{I}\end{array}$ & $\begin{array}{l}\mathrm{C} \\
\mathrm{T} \\
\mathrm{C} \\
\mathrm{G} \\
\mathrm{G} \\
\mathrm{A}\end{array}$ & $\begin{array}{l}\mathrm{T} \\
\mathrm{C} \\
\mathrm{T} \\
\mathrm{A} \\
\mathrm{A} \\
\mathrm{G}\end{array}$ & $\begin{array}{l}\mathrm{A} \\
\mathrm{G}\end{array}$ & $\begin{array}{l}\text { Yes } \\
\text { Yes } \\
\text { Yes } \\
\text { Yes } \\
\text { Yes } \\
\text { Yes }\end{array}$ \\
\hline
\end{tabular}

Notes: ${ }^{a}$ Conservation of amino acids in polypeptides or nucleotides in RNA in Human $(H)$, cows (B), mice (M) and Xenopus laevis $(X) .{ }^{b}$ rCRS: revised Cambridge reference

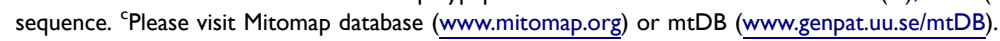

located at processing site for the tRNA $5^{\prime}$ end precursors, catalyzed by RNase P. ${ }^{53}$ Furthermore, A5655G variant changed the highly conserved base pairing (A1-U72) at the Acceptor arm of tRNA ${ }^{\text {Ala }}$. Functional analysis revealed that this variant caused an improperly aminoacylated tRNA $^{\text {Ala }}$ and slower electrophoretic mobility of mutated tRNA. ${ }^{54}$ While the homoplasmic T5587C variant occurred at position 73 near the end of tRNA ${ }^{\text {Ala }}$, which was very important for tRNA identity. ${ }^{55}$ Notably, the $\mathrm{T}$ to $\mathrm{C}$ transition at that position was extremely conserved from various species, recent experimental studies indicated that the
T5587C variant may be associated with Leber's Hereditary Optic Neuropathy (LHON) and essential hypertension (EH). ${ }^{56,57}$ Furthermore, the $\mathrm{C} 7502 \mathrm{~T}$ and $\mathrm{T} 7505 \mathrm{C}$ variants were identified in DHU-loop of tRNA ${ }^{\text {Ser(UCN) }}$ gene, the C7502T variant occurred at A14-U8 interaction site, which is important for cognate aminoacyl tRNA synthetase recognition. ${ }^{58}$ While the $\mathrm{T} 7505 \mathrm{C}$ variant was located at a highly conserved base-pairing (A10-U20) of tRNA $^{\text {Ser(UCN) }}$. The abolishment of A10-U20 Watson-Crick base-pairing likely altered the tRNA ${ }^{\mathrm{Ser}(\mathrm{UCN})}$ metabolism. Functional significant of this variant was supported by 

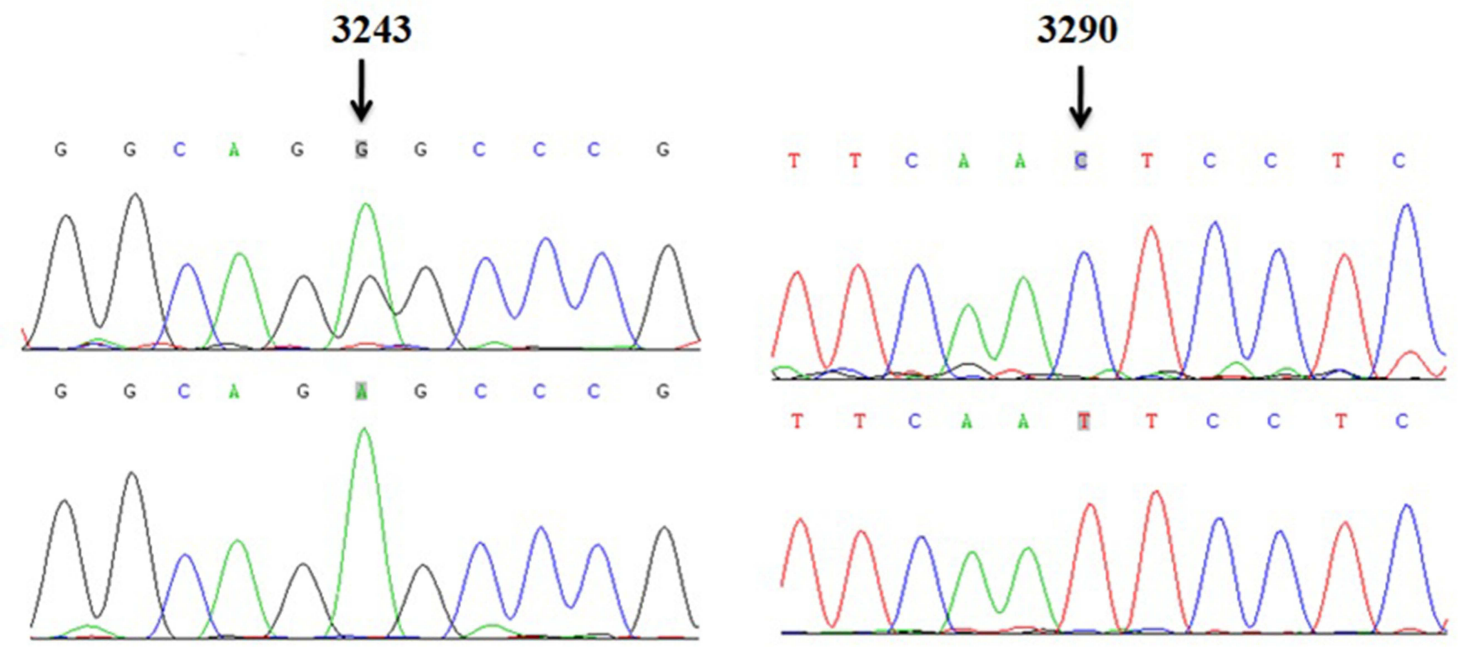

Mutant

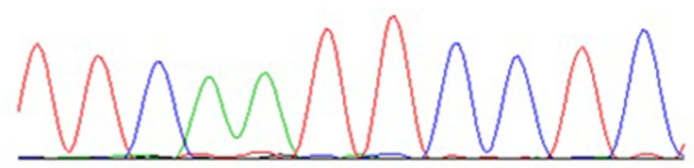

Control

Figure 3 Identification of tRNA ${ }^{\text {Leu(UUR) }}$ A3243G and T3290C variants by direct sequence.

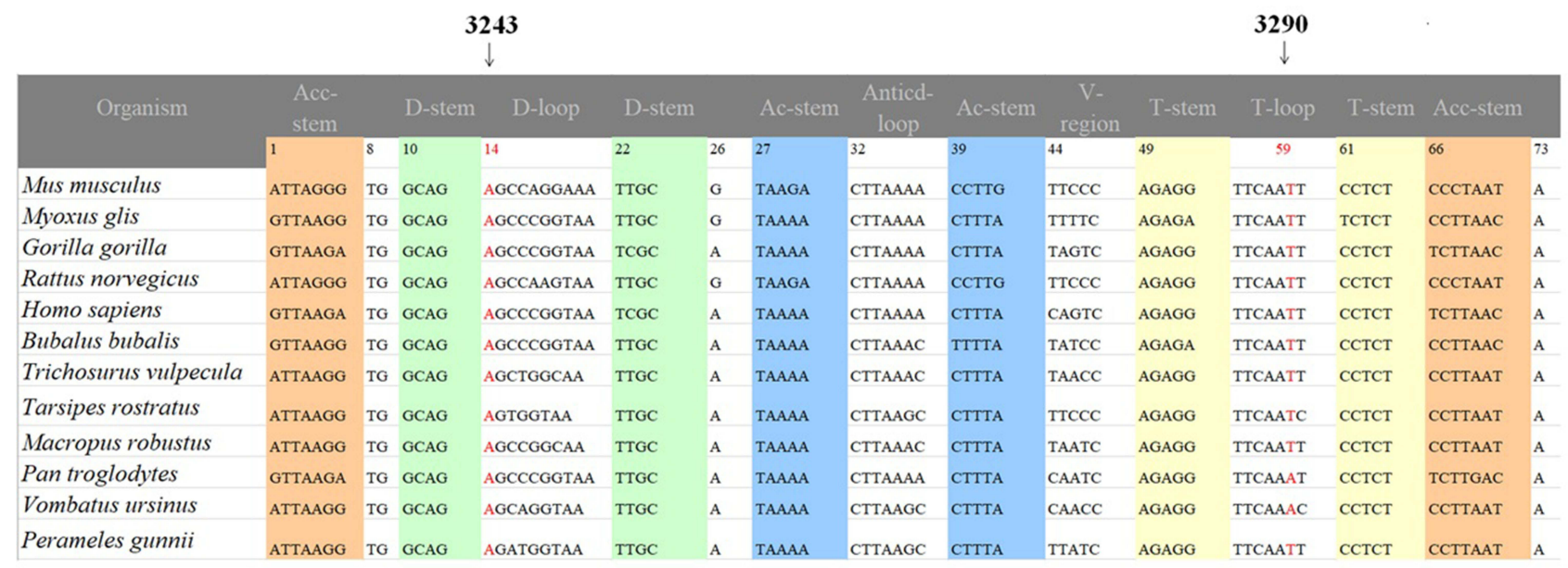

Figure 4 Sequence alignment of tRNA ${ }^{\text {Leu(UUR) }}$ from various species, arrows indicate the positions of 14 and 59 , corresponding to the A3243G and T3290C variants.

approximately $65 \%$ reductions in the level of tRNA ${ }^{\text {Ser(UCN) }}$ observed in the lymphoblastoid cell lines carrying the T7505C variant, as compared with the wild-type cell lines. ${ }^{59}$ In addition, the heteroplasmic G8313A variant had been found in patients with various clinical phenotypes including encephaloneuropathy, short stature, myopathy, peripheral neuropathy, and osteoporosis. ${ }^{60}$ Functional analysis of cybrid cells containing this variant showed a marked decreased in tRNA ${ }^{\text {Lys }}$ steady-state level and aminoacylation ability, suggesting that these molecular abnormalities may underlie the pathogenesis of the G8313A variant. ${ }^{61}$ Moreover, the $\mathrm{T} 10003 \mathrm{C}$ and $\mathrm{A} 10055 \mathrm{G}$ variants were identified in $\mathrm{tRNA}^{\mathrm{Gly}}$ gene, in fact, the $\mathrm{T} 10003 \mathrm{C}$ variant created a novel Watson-Crick base-pairing (C13G19), whereas the A10055G variant disrupted the conserved Watson-Crick base-pairing (U3-A70), therefore, the alteration of mt-tRNA structure caused by A10055G variant may affect mt-tRNA function, and subsequently led to a failure in mt-tRNA metabolism. ${ }^{62}$ Moreover, the A12308G variant created a novel Watson-Crick basepairing (A25-U43) in the variable region of tRNA ${ }^{\mathrm{Leu}(\mathrm{CUN})}$, by contrast, the A12330G variant abolished the conserved base-pairing (U6-A68) in the Acceptor arm of tRNA $^{\text {Leu(CUN) }}$. In fact, the A12308G variant had been implicated to be associated with pigmentary retinal degeneration, short stature, dysphasia-dysarthria and cardiac conduction defects, ${ }^{63,64}$ and increased the risk of developing stroke. ${ }^{65}$ While the homoplasmic A12330G variant was found in patients with maternally inherited $\mathrm{EH}^{66}$ Furthermore, 3 variants were found in $\mathrm{RRNA}^{\mathrm{Thr}}$ gene: 
G15897A, A15924G and G15927A, among these, the G15897A variant occurred at highly conserved nucleotide of RNAA $^{\text {Thr }}$, which was important for the stability and identity of mt-tRNA. This variant was found to decrease the steady-state level, as well as aminoacylation ability of tRNA $^{\text {Thr }}$, and subsequently led to the defects in energetic processes. ${ }^{11}$ Notably, the A15924G and G15927A variants were located at anticodon stem of tRNA ${ }^{\text {Thr }}$, which were extremely conserved from different species. ${ }^{67}$ Previous studies showed that the A15924G variant was associated with fatal infantile respiratory enzyme deficiency, ${ }^{68}$ while the G15927A was regarded as pathogenic mutation associated with coronary heart disease $(\mathrm{CHD})^{69}$ and implicated to modulate the clinical expression of deafness-associated 12S rRNA A1555G mutation. ${ }^{70}$ The G15927A mutation caused significantly decreased efficiency in aminoacylation and steady-state levels of $\mathrm{tRNA}^{\mathrm{Thr}}{ }^{71}$ Thus, the failure in tRNA metabolism led to the mitochondrial dysfunction that was responsible for T2DM.

Among these patients carrying putative pathogenic mttRNA variants, only 2 of them had an obvious family history of T2DM (Figure 1). In DM-101 pedigree, among 6 matrilineal relatives, 3 individuals suffered from both diabetes and deafness, while in DM-102 pedigree, there were 8 matrilineal relatives, notably, 4 of them suffered from T2DM (2 subjects with diabetes only, 2 subjects with both diabetes and hearing impairment). Interestingly, matrilineal relatives in these two families had earlier age onset of diabetes, indicating that mtDNA variants may be acted as risk factors for molecular diagnosis and detection of this disease.

Sequence analysis of the entire mitochondrial genomes of the matrilineal relatives from two families revealed the presence of tRNA $^{\text {Leu(UUR) }}$ A3243G and T3290C variants, together with sets of genetic polymorphisms belonging to East Asian haplogroups F2 and D4, respectively. ${ }^{29}$ In fact, the heteroplasmic A3243G mutation accounted for approximately $0.5-3 \%$ of all cases of diabetes in general population. ${ }^{72,73}$ Patients who carried this mutation demonstrated an impaired OXPHOS, ${ }^{74}$ and decreased OXPHOS in glucose-stimulated insulin secretion from $\beta$-cells. ${ }^{75}$ On the other hand, the homoplasmic T3290C variant occurred at position 59 in the T $\psi \mathrm{C}$ loop of tRNA ${ }^{\mathrm{Leu(UUR})}$, which was very conserved from different species (Figure 4). Thus, it was hypothesized that T3290C variant likely altered the tRNA ${ }^{\text {Leu(UUR) }}$ metabolism, in fact, previous study suggested that this variant was found to be associated with $\mathrm{EH}^{46}$ Moreover, a variable severity of $\mathrm{T} 2 \mathrm{DM}$, as well as audiometric configuration of hearing impairment were observed among the matrilineal relatives in these two families indicated the involvement of other modified factors such as nuclear genes, mitochondrial haplogroups, epigenetic or environmental factors. However, the absence of TRMU A10S and GJB2 variants indicated that these nuclear modified genes may not play active roles in MIDD in these families. Furthermore, sequence analysis of the entire mtDNA genes of matrilineal relatives in these families suggested the presence of 74 variants, in addition to the A3243G and T3290C variants, which belonged to mitochondrial haplogroups F2 and D4, respectively. ${ }^{29}$ Of these, the ND1 T3394C variant displayed very highly evolutionary conservation, and was not detected in 200 Chinese control subjects. Notably, this variant disrupted the specific electrostatic interactions between $\mathrm{Y} 30$ of $N D 1$ with the sidechain of E4 and backbone carbonyl group of M1 of NADH dehydrogenase [ubiquinone] 1 alpha subcomplex subunit 1 (NDUFA1) of Complex I, thereby altering the structure and function of OXPHOS. ${ }^{76}$ Importantly, the T3394C variant altered the stability of $N D 1$ and Complex I assembly, decreased the activities of Complex I and increased the production of ROS in cybrid cells. ${ }^{77}$ Therefore, the mitochondrial dysfunctions caused by the tRNA ${ }^{\mathrm{Leu}(U U R)}$ T3290C variant may be worsened by the ND1 T3394C variant in DM-102 pedigree.

\section{Conclusions}

In summary, our study indicated that mt-tRNA variants may be associated with T2DM in Han Chinese population, in particular, tRNA ${ }^{\mathrm{Leu}(U U R)} \mathrm{A} 3243 \mathrm{G}$ and T3290C, tRNA $^{\text {Met }}$ A4435G and C4467A, tRNA ${ }^{\text {Trp }}$ A5514G, tRNA $^{\text {Ala }}$ T5587C and A5655G, tRNA ${ }^{\text {Ser(UCN) }}$ C7502T and $\mathrm{T} 7505 \mathrm{C}$, tRNA ${ }^{\text {Lys }}$ G8313A, tRNA ${ }^{\text {Gly }}$ T10003C and A10055G, tRNA ${ }^{\text {Leu(CUN) }}$ A12308G and A12330G, tRNA $^{\text {Thr }}$ G15897A, A15924G and G15927A altered the structure and function of their tRNAs, thereby causing mitochondrial dysfunctions and long-standing increase of ROS in pancreatic $\beta$-cells. These variants may be the inherited risk factors for T2DM. Future studies with larger sample size comprising different ethnicities should be performed to confirm our conclusion.

\section{Ethics Approval and Consent to Participate}

This study was conducted with approval from the Ethics Committee of the Yantai Affiliated Hospital of Binzhou 
Medical University (No. 20210212001). This study was conducted in accordance with the declaration of Helsinki. Written informed consent was obtained from all participants.

\section{Funding}

There is no funding to report.

\section{Disclosure}

The authors declare that they have no conflicts of interest for this work.

\section{References}

1. Meetoo D, McGovern P, Safadi R. An epidemiological overview of diabetes across the world. Br J Nurs. 2007;16(16):1002-1007. doi:10.12968/bjon.2007.16.16.27079

2. Owen KR, McCarthy MI. Genetics of type 2 diabetes. Curr Opin Genet Dev. 2007;17:239-244. doi:10.1016/j.gde.2007.04.003

3. Maassen JA, Hart LM, Van Essen E, et al. Mitochondrial diabetes: molecular mechanisms and clinical presentation. Diabetes. 2004;53: S103-109. doi:10.2337/diabetes.53.2007.S103

4. Ballinger SW, Shoffner JM, Hedaya EV, et al. Maternally transmitted diabetes and deafness associated with a $10.4 \mathrm{~kb}$ mitochondrial DNA deletion. Nat Genet. 1992;1:11-15. doi:10.1038/ng0492-11

5. Stowe DF, Camara AK. Mitochondrial reactive oxygen species production in excitable cells: modulators of mitochondrial and cell function. Antioxid Redox Signal. 2009;11:1373-1414. doi:10.1089/ ars.2008.2331

6. Taanman JW. The mitochondrial genome: structure, transcription, translation and replication. Biochim Biophys Acta. 1999;14 10:103-123. doi:10.1016/S0005-2728(98)00161-3

7. van den Ouweland JM, Lemkes HH, Ruitenbeek W, et al. Mutation in mitochondrial tRNA(Leu)(UUR) gene in a large pedigree with maternally transmitted type II diabetes mellitus and deafness. Nat Genet. 1992;1:368-371. doi:10.1038/ng0892-368

8. Suzuki Y, Suzuki S, Hinokio Y, et al. Diabetes associated with a novel 3264 mitochondrial tRNA(Leu)(UUR) mutation. Diabetes Care. 1997;20:1138-1140. doi:10.2337/diacare.20.7.1138

9. Li W, Wen $\mathrm{C}$, Li W, et al. The tRNA(Gly) T10003C mutation in mitochondrial haplogroup $\mathrm{M} 11 \mathrm{~b}$ in a Chinese family with diabetes decreases the steady-state level of tRNA(Gly), increases aberrant reactive oxygen species production, and reduces mitochondrial membrane potential. Mol Cell Biochem. 2015;408:171-179. doi:10.1007/ s11010-015-2493-0

10. Mezghani N, Mkaouar-Rebai E, Mnif M, et al. The heteroplasmic m.14709T $>$ C mutation in the tRNA(Glu) gene in two Tunisian families with mitochondrial diabetes. $J$ Diabetes Complications. 2010;24:270-277. doi:10.1016/j.jdiacomp.2009.11.002

11. Li K, Wu L, Liu J, et al. Maternally inherited diabetes mellitus associated with a novel m.15897G $>$ A mutation in mitochondrial tRNAThr gene. J Diabetes Res. 2020;2020:2057187. doi:10.1155/ 2020/2057187

12. Rossmanith W, Karwan RM. Impairment of tRNA processing by point mutations in mitochondrial tRNALeu(UUR) associated with mitochondrial diseases. FEBS Lett. 1998;433:269-274. doi:10.1016/ S0014-5793(98)00928-4

13. Yasukawa T, Suzuki T, Ueda T, et al. Modification defect at anticodon wobble nucleotide of mitochondrial tRNAsLeu(UUR) with pathogenic mutations of mitochondrial myopathy, encephalopathy, lactic acidosis, and stroke-like episodes. $J$ Biol Chem. 2000;275:4251-4257. doi:10.1074/jbc.275.6.4251
14. El Meziane A, Lehtinen SK, Hance N, et al. A tRNA suppressor mutation in human mitochondria. Nat Genet. 1998;18:350-353. doi:10.1038/ng0498-350

15. Liu G, Shen X, Sun Y, et al. Heteroplasmy and phenotype spectrum of the mitochondrial tRNALeu(UUR) gene m.3243A $>\mathrm{G}$ mutation in seven Han Chinese families. J Neurol Sci. 2020;408:116562. doi:10.1016/j.jns.2019.116562

16. American Diabetes Association. Diagnosis and classification of diabetes mellitus. Diabetes Care. 2010;33:S62-S69. doi:10.2337/dc10S062

17. Xue L, Wang M, Li H, et al. Mitochondrial tRNA mutations in 2070 Chinese Han subjects with hypertension. Mitochondrion. 2016;30:208-221. doi:10.1016/j.mito.2016.08.008

18. Ying Z, Zheng J, Cai Z, et al. Mitochondrial haplogroup B increases the risk for hearing loss among the Eastern Asian pedigrees carrying 12S rRNA 1555A > G mutation. Protein Cell. 2015;6:844-848. doi:10.1007/s13238-015-0203-z

19. Rieder MJ, Taylor SL, Tobe VO, et al. Automating the identification of DNA variations using quality-based fluorescence resequencing: analysis of the human mitochondrial genome. Nucleic Acids Res. 1998;26:967-973. doi:10.1093/nar/26.4.967

20. Andrews RM, Kubacka I, Chinnery PF, et al. Reanalysis and revision of the Cambridge reference sequence for human mitochondrial DNA. Nat Genet. 1999;23(2):147. doi:10.1038/13779

21. Ding Y, Teng YS, Zhuo GC, et al. The mitochondrial tRNAHis G12192A mutation may modulate the clinical expression of deafness-associated tRNAThr G15927A mutation in a Chinese pedigree. Curr Mol Med. 2019;19:136-146. doi:10.2174/156652 4019666190308121552

22. Levin L, Zhidkov I, Gurman Y, et al. Functional recurrent mutations in the human mitochondrial phylogeny: dual roles in evolution and disease. Genome Biol Evol. 2013;5(5):876-890. doi:10.1093/gbe/evt058

23. Florentz C, Sohm B, Tryoen-Toth P, et al. Human mitochondrial tRNAs in health and disease. Cell Mol Life Sci. 2003;60:1356-1375. doi:10.1007/s00018-003-2343-1

24. Sprinzl M, Vassilenko KS. Compilation of tRNA sequences and sequences of tRNA genes. Nucleic Acids Res. 2005;33:D139-40. doi:10.1093/nar/gki012

25. Ding Y, Xia BH, Liu Q, et al. Allele-specific PCR for detecting the deafness-associated mitochondrial 12S rRNA mutations. Gene. 2016;591:148-152. doi:10.1016/j.gene.2016.07.013

26. Huang S, Gao X, Wang Y, et al. Prelingual sensorineural hearing loss caused by a novel GJB2 dominant mutation in a Chinese family. Biomed Res Int. 2020;2020:6370386.

27. Joint National Committee on Prevention, Detection, Evaluation and Treatment of High Blood Pressure. The sixth report of the Joint National Committee on Prevention, Detection, Evaluation and Treatment of High Blood Pressure. Arch Intern Med. 1997;157:2413-2446. doi:10.1001/archinte.1997.00440420033005

28. Wu L, Li R, Chen J, et al. Analysis of mitochondrial A1555G mutation in infants with hearing impairment. Exp Ther Med. 2018;15:5307-5313

29. Kong QP, Bandelt HJ, Sun C, et al. Updating the East Asian mtDNA phylogeny: a prerequisite for the identification of pathogenic mutations. Hum Mol Genet. 2006;15:2076-2086. doi:10.1093/hmg/ddl130

30. Zhang J, Lu B, Xia WW, et al. The mitochondrial transfer RNAAsp A7551G mutation may contribute to the clinical expression of deafness associated with the A1555G mutation in a pedigree with hearing impairment. Mol Med Rep. 2019;19:1797-1802.

31. Guan MX, Yan Q, Li X, et al. Mutation in TRMU related to transfer RNA modification modulates the phenotypic expression of the deafness-associated mitochondrial $12 \mathrm{~S}$ ribosomal RNA mutations. Am J Hum Genet. 2006;79:291-302. doi:10.1086/506389

32. Ruiz-Pesini E, Wallace DC. Evidence for adaptive selection acting on the tRNA and rRNA genes of human mitochondrial DNA. Hum Mutat. 2006;27:1072-1081. doi:10.1002/humu.20378 
33. Liang M, Guan M, Zhao F, et al. Leber's hereditary optic neuropathy is associated with mitochondrial ND1 T3394C mutation. Biochem Biophys Res Commun. 2009;383:286-292. doi:10.1016/j.bbrc.2009.03.097

34. Im I, Jang MJ, Park SJ, et al. Mitochondrial respiratory defect causes dysfunctional lactate turnover via AMP-activated protein kinase activation in human-induced pluripotent stem cell-derived hepatocytes. J Biol Chem. 2015;290:29493-29505. doi:10.1074/jbc.M115.670364

35. Liu XL, Zhou X, Zhou J, et al. Leber's hereditary optic neuropathy is associated with the T12338C mutation in mitochondrial ND5 gene in six Han Chinese families. Ophthalmology. 2011;118:978-985. doi:10.1016/j.ophtha.2010.09.003

36. van Oven M, Kayser M. Updated comprehensive phylogenetic tree of global human mitochondrial DNA variation. Hum Mutat. 2009;30: E386-394. doi:10.1002/humu.20921

37. Bibb MJ, Van Etten RA, Wright CT, et al. Sequence and gene organization of mouse mitochondrial DNA. Cell. 1981;26 (2):167-180. doi:10.1016/0092-8674(81)90300-7

38. Gadaleta G, Pepe G, De Candia G, et al. The complete nucleotide sequence of the Rattus norvegicus mitochondrial genome: cryptic signals revealed by comparative analysis between vertebrates. $J \mathrm{Mol}$ Evol. 1989;28:497-516. doi:10.1007/BF02602930

39. Roe BA, Ma DP, Wilson RK, et al. The complete nucleotide sequence of the Xenopus laevis mitochondrial genome. J Biol Chem. 1985;260:9759-9774. doi:10.1016/S0021-9258(17)39303-1

40. Chan DK, Chang KW. GJB2-associated hearing loss: systematic review of worldwide prevalence, genotype, and auditory phenotype. Laryngoscope. 2014;124:E34-E53. doi:10.1002/lary.24332

41. Meng F, Cang X, Peng Y, et al. Biochemical evidence for a nuclear modifier allele (A10S) in TRMU (Methylaminomethyl-2-thiouridylatemethyltransferase) related to mitochondrial tRNA modification in the phenotypic manifestation of deafness-associated 12S rRNA mutation. J Biol Chem. 2017;292:2881-2892. doi:10.1074/jbc.M116.749374

42. Maassen JA, 'T Hart LM, van Essen E. Mitochondrial diabetes: molecular mechanisms and clinical presentation. Diabetes. 2004;53 (Supplement 1):S103-109.

43. Yu N, Zhang YF, Zhang K, et al. MELAS and Kearns-Sayre overlap syndrome due to the mtDNA m.A3243G mutation and large-scale mtDNA deletions. eNeurological Sci. 2016;4:15-18. doi:10.1016/j. ensci.2016.04.006

44. Chomyn A, Enriquez JA, Micol V, et al. The mitochondrial myopathy, encephalopathy, lactic acidosis, and stroke-like episode syndrome-associated human mitochondrial tRNALeu(UUR) mutation causes aminoacylation deficiency and concomitant reduced association of mRNA with ribosomes. J Biol Chem. 2000;275(25):19 198-19209. doi:10.1074/jbc.M908734199

45. Janssen GM, Maassen JA. The diabetes-associated 3243 mutation in the mitochondrial tRNA(Leu(UUR)) gene causes severe mitochondrial dysfunction without a strong decrease in protein synthesis rate. J Biol Chem. 1999;274:29744-29748. doi:10.1074/jbc.274.42.29 744

46. Zhu HY, Wang SW, Liu L, et al. Genetic variants in mitochondrial tRNA genes are associated with essential hypertension in a Chinese Han population. Clin Chim Acta. 2009;410:64-69. doi:10.1016/j. cca.2009.09.023

47. Lu Z, Chen H, Meng Y, et al. The tRNAMet $4435 \mathrm{~A}>\mathrm{G}$ mutation in the mitochondrial haplogroup G2a1 is responsible for maternally inherited hypertension in a Chinese pedigree. Eur J Hum Genet. 2011;19:1181-1186. doi:10.1038/ejhg.2011.111

48. Zhou M, Xue L, Chen Y, et al. A hypertension-associated mitochondrial DNA mutation introduces an m1G37 modification into tRNAMet, altering its structure and function. $J$ Biol Chem. 2018;293:1425-1438. doi:10.1074/jbc.RA117.000317

49. Liu Y, Li Y, Zhu C, et al. Mitochondrial biogenesis dysfunction and metabolic dysfunction from a novel mitochondrial tRNAMet $4467 \mathrm{C}>\mathrm{A}$ mutation in a Han Chinese family with maternally inherited hypertension. Sci Rep. 2017;7:3034. doi:10.1038/s41598-017-03303-w
50. Brzezniak LK, Bijata M, Szczesny RJ, et al. Involvement of human ELAC2 gene product in $3^{\prime}$ end processing of mitochondrial tRNAs. RNA Biol. 2011;8:616-626. doi:10.4161/rna.8.4.15393

51. Wilson FH, Hariri A, Farhi A, et al. A cluster of metabolic defects caused by mutation in a mitochondrial tRNA. Science. 2004;306:1190-1194. doi:10.1126/science.1102521

52. Del Mar O'Callaghan M, Emperador S, López-Gallardo E, et al. New mitochondrial DNA mutations in tRNA associated with three severe encephalopamyopathic phenotypes: neonatal, infantile, and childhood onset. Neurogenetics. 2012;13:245-250. doi:10.1007/s10048-012-0322-0

53. Holzmann J, Frank P, Löffler E, et al. RNase P without RNA: identification and functional reconstitution of the human mitochondrial tRNA processing enzyme. Cell. 2008;135(3):462-474. doi:10.1016/j.cell.2008.09.013

54. Jiang P, Wang M, Xue L, et al. A hypertension-associated tRNAAla mutation alters tRNA metabolism and mitochondrial function. $\mathrm{Mol}$ Cell Biol. 2016;36:1920-1930. doi:10.1128/MCB.00199-16

55. Levinger L, Mörl M, Florentz C. Mitochondrial tRNA 3' end metabolism and human disease. Nucleic Acids Res. 2004;32:5430-5441. doi:10.1093/nar/gkh884

56. Ji Y, Qiao L, Liang X, et al. Leber's hereditary optic neuropathy is potentially associated with a novel $\mathrm{m} .5587 \mathrm{~T}>\mathrm{C}$ mutation in two pedigrees. Mol Med Rep. 2017;16:8997-9004. doi:10.3892/mmr.2017.7734

57. Lin L, Cui P, Qiu Z, et al. The mitochondrial tRNAAla 5587T $>\mathrm{C}$ and tRNALeu(CUN) $12280 \mathrm{~A}>\mathrm{G}$ mutations may be associated with hypertension in a Chinese family. Exp Ther Med. 2019;17: $1855-1862$.

58. Jiang P, Ling Y, Zhu T, et al. Mitochondrial tRNA mutations in Chinese children with Tic disorders. Biosci Rep. 2020;40: BSR20201856. doi:10.1042/BSR20201856

59. Tang X, Li R, Zheng J, et al. Maternally inherited hearing loss is associated with the novel mitochondrial tRNA Ser(UCN) 7505T $>C$ mutation in a Han Chinese family. Mol Genet Metab. 2010;100:57-64. doi:10.1016/j.ymgme.2010.01.008

60. O'Rourke K, Buddles MR, Farrell M, et al. Phenotypic diversity associated with the mitochondrial $\mathrm{m} .8313 \mathrm{G}>\mathrm{A}$ point mutation. Muscle Nerve. 2009;40:648-651. doi:10.1002/mus.21342

61. Bacman SR, Atencio DP, Moraes CT. Decreased mitochondrial tRNALys steady-state levels and aminoacylation are associated with the pathogenic G8313A mitochondrial DNA mutation. Biochem J. 2003;374:131-136. doi:10.1042/bj20030222

62. Liu H, Li R, Li W, et al. Maternally inherited diabetes is associated with a homoplasmic $\mathrm{T} 10003 \mathrm{C}$ mutation in the mitochondrial tRNA(Gly) gene. Mitochondrion. 2015;21:49-57. doi:10.1016/j. mito.2015.01.004

63. Crimi M, Del Bo R, Galbiati S, et al. Mitochondrial A12308G polymorphism affects clinical features in patients with single mtDNA macrodeletion. Eur J Hum Genet. 2003;11:896-898. doi:10.1038/sj.ejhg. 5201056

64. Zifa E, Theotokis P, Kaminari A, et al. A novel G3337A mitochondrial ND1 mutation related to cardiomyopathy co-segregates with tRNALeu(CUN) A12308G and tRNAThr C15946T mutations. Mitochondrion. 2008;8:229-236. doi:10.1016/j.mito.2008.04.001

65. Pulkes T, Sweeney MG, Hanna MG. Increased risk of stroke in patients with the A12308G polymorphism in mitochondria. Lancet. 2000;356:2068-2069. doi:10.1016/S0140-6736(00)03408-5

66. Teng L, Zheng J, Leng J, et al. Clinical and molecular characterization of a Han Chinese family with high penetrance of essential hypertension. Mitochondrial DNA. 2012;23(6):461-465. doi:10.31 09/19401736.2012.710205

67. Helm M, Brulé H, Friede D, et al. Search for characteristic structural features of mammalian mitochondrial tRNAs. RNA. 2000;6: 1356-1379. doi:10.1017/S1355838200001047

68. Yoon KL, Aprille JR, Ernst SG. Mitochondrial tRNA(thr) mutation in fatal infantile respiratory enzyme deficiency. Biochem Biophys Res Commun. 1991;176:1112-1115. doi:10.1016/0006-291X(91)90399-R 
69. Jia Z, Wang X, Qin Y, et al. Coronary heart disease is associated with a mutation in mitochondrial tRNA. Hum Mol Genet. 2013;22: 4064-4073. doi:10.1093/hmg/ddt256

70. Wang X, Lu J, Zhu Y, et al. Mitochondrial tRNAThr G15927A mutation may modulate the phenotypic manifestation of ototoxic 12S rRNA A1555G mutation in four Chinese families. Pharmacogenet Genomics. 2008;18:1059-1070. doi:10.1097/FPC.0b013e3283131661

71. Jia Z, Zhang Y, Li Q, et al. A coronary artery disease-associated tRNAThr mutation altered mitochondrial function, apoptosis and angiogenesis. Nucleic Acids Res. 2019;47:2056-2074.

72. Ohkubo K, Yamano A, Nagashima M, et al. Mitochondrial gene mutations in the tRNA(Leu(UUR)) region and diabetes: prevalence and clinical phenotypes in Japan. Clin Chem. 2001;47:1641-1648. doi:10.1093/clinchem/47.9.1641

73. Walker M, Taylor RW, Turnbull DM. Mitochondrial diabetes. Diabet Med. 2005;22:18-20. doi:10.1111/j.1464-5491.2005.1761f.x
74. Liao WQ, Pang Y, Yu CA, et al. Novel mutations found in mitochondrial diabetes in Chinese Han population. Diabetes Res Clin Pract. 2007;76:425-435. doi:10.1016/j.diabres.2006.09.032

75. Gerbitz KD, Gempel K, Brdiczka D. Mitochondria and diabetes. Genetic, biochemical, and clinical implications of the cellular energy circuit. Diabete. 1996;45:113-126. doi:10.2337/diab.45.2. 113

76. Zhu J, Vinothkumar KR, Hirst J. Structure of mammalian respiratory complex I. Nature. 2016;536:354-358. doi:10.1038/ nature 19095

77. Ji Y, Zhang J, Yu J, et al. Contribution of mitochondrial ND1 $3394 \mathrm{~T}>\mathrm{C}$ mutation to the phenotypic manifestation of Leber's hereditary optic neuropathy. Hum Mol Genet. 2019;28:1515-1529. doi:10.1093/hmg/ddy450

\section{Publish your work in this journal}

The International Journal of General Medicine is an international, peer-reviewed open-access journal that focuses on general and internal medicine, pathogenesis, epidemiology, diagnosis, monitoring and treatment protocols. The journal is characterized by the rapid reporting of reviews, original research and clinical studies across all disease areas. The manuscript management system is completely online and includes a very quick and fair peer-review system, which is all easy to use. Visit http://www.dovepress.com/ testimonials.php to read real quotes from published authors. 